\title{
A Novel Image Compression Method Based on Classified Energy and Pattern Building Blocks
}

\author{
Umit Guz \\ Department of Electrical-Electronics Engineering, Engineering Faculty, Isik University, Sile, 34980 Istanbul, Turkey \\ Correspondence should be addressed to Umit Guz, guz@isikun.edu.tr
}

Received 26 August 2010; Revised 23 January 2011; Accepted 9 February 2011

Academic Editor: Karen Panetta

Copyright () 2011 Umit Guz. This is an open access article distributed under the Creative Commons Attribution License, which permits unrestricted use, distribution, and reproduction in any medium, provided the original work is properly cited.

In this paper, a novel image compression method based on generation of the so-called classified energy and pattern blocks (CEPB) is introduced and evaluation results are presented. The CEPB is constructed using the training images and then located at both the transmitter and receiver sides of the communication system. Then the energy and pattern blocks of input images to be reconstructed are determined by the same way in the construction of the CEPB. This process is also associated with a matching procedure to determine the index numbers of the classified energy and pattern blocks in the CEPB which best represents (matches) the energy and pattern blocks of the input images. Encoding parameters are block scaling coefficient and index numbers of energy and pattern blocks determined for each block of the input images. These parameters are sent from the transmitter part to the receiver part and the classified energy and pattern blocks associated with the index numbers are pulled from the CEPB. Then the input image is reconstructed block by block in the receiver part using a mathematical model that is proposed. Evaluation results show that the method provides considerable image compression ratios and image quality even at low bit rates.

\section{Introduction}

Raw or uncompressed multimedia data such as graphics, still images, audio, and video requires substantial storage capacity and transmission bandwidth. The recent growth of data intensive multimedia-based applications has not only maintained the need for more efficient ways to encode the audio signals and images but also have required high compression ratio and fast communication technology [1].

At the present state of the technology in order to overcome some limitations on storage, transmission bandwidth, and transmission time, the images must be compressed before their storage and transmission and decompressed at the receiver part [2].

Especially uniform or plain areas in the still images contain adjacent picture elements (pixels) which have almost the same numeric values. This case results in large number of spatial redundancy (or correlation between pixel values which numerically close to each other) and highly correlated regions in the images $[3,4]$. The idea behind the compression is to remove this redundancy in order to get more efficient ways to represent the still images. The performance of the compression algorithm is measured by the compression ratio (CR) and it is defined as a ratio between the original image data size and compressed image data size. In general, the compression algorithms can be grouped as lossy and lossless compression algorithms. It is very well known that, in the lossy compression schemes, the image compression algorithm should achieve a tradeoff between the image quality and the compression ratio [5]. It should be noted that, higher compression ratios produce lower image quality and the image quality can be effected by the other characteristics, some details or content of the input image.

Image compression techniques with different schemes have been developed especially since 1990s. These techniques are generally based on Discrete Cosine Transform (DCT), Wavelet Transform and the other transform domain techniques such as Principal Component Analysis (PCA) or Karhunen-Loève Decomposition (KLD) [6-8]. Transform domain techniques are widely used methods to compress the still images. The compression performance of these methods is affected by several factors such as block size, entropy, quantization error, truncation error and coding gain. In these methods, two-dimensional images are transformed 
from the spatial domain to the frequency domain. It is proved that, the human visual system (HVS) is more sensitive to energy with low spatial frequency than with high spatial frequency. While the low spatial frequency components correspond to important image features, the high frequency ones correspond to image details. Therefore, compression can be achieved by quantizing and transmitted the most important or low-frequency coefficients while the remaining coefficients are discarded. The standards for compression of still images such as JPEG [9-11] exploit the DCT, which represents a still image as a superposition of cosine functions with different discrete frequencies [12]. The transformed image data is represented as a function of two spatial dimensions, and its components are called spatial frequencies or DCT coefficients. First, the image data is divided into $N \times N$ blocks and each block is transformed independently to obtain $N \times N$ coefficients. Some of the DCT coefficients computed for the image blocks will be close to zero. In order to reduce the quantization levels, these coefficients are set to zero and the remaining coefficients are represented with reduced precision or fewer bits. After this process the quantization results in loss of information but it also provides the compression.

The usage of uniformly sized image blocks simplifies the compression, but it does not take into account the irregular regions within the real images. The fundamental limitation of the DCT-based compression is the block-based segmentation or framing [13]. In these methods, depend on the block size of the images, the degradation which is also known as the "blocking effect" occurs. A larger block leads to more efficient coding or compression but requires more computational power. Although image degradation is noticeable especially when large DCT blocks are used, the compression ratio is higher. Therefore, most existing systems use image blocks of $8 \times 8$ or $16 \times 16$ pixels as a compromise between coding or compression efficiency and image quality.

Recently, there are too many works on image coding that have been focused on the Discrete Wavelet Transform (DWT). Because of its data reduction capability, DWT has become a standard method in the image compression applications. In the wavelet compression, the image data is transformed and compressed as a single data object rather than block by block as in a DCT-based compression. In wavelet compression a uniform distribution of compression error occurs across the image. DWT provides an adaptive spatial-frequency resolution which is well suited to the properties of an HVS. In other words, DWT provides better spatial resolution at high frequencies and better frequency resolution at low frequencies. It also offers better image quality than DCT, especially on a higher compression ratio [14]. However, the implementation or computational complexity of the DWT is more expensive than that of the DCT.

Wavelet transform (WT) represents an image as a sum of wavelet functions (wavelets) with different locations and scales [15]. Decomposition of an image into the wavelets involves a pair of waveforms. One of the waveform represents the high frequencies corresponding to the detailed parts of an image called wavelet function and the other one represents the low frequencies or smooth parts of an image called scaling function.

A wide variety of wavelet-based image compression schemes have been proposed in the literature [16]. The early wavelet image coders [17-19] were designed to exploit the ability of compacting energy on the wavelet decomposition. The advantages of the wavelet coders with respect to DCT based ones were quantizers and variable length entropy coders that they used. Subsequent works were focused on exploiting the wavelet coefficients more efficiently. In this manner, Shapiro [20] developed a wavelet-based encoder, called Embedded Zero-tree Wavelet encoder (EZW). Usage of zero trees in EZW encoder showed that coding the wavelet coefficients efficiently can lead to image compression schemes that are fast and effective by means of ratedistortion performance. Said and Pearlman [21] proposed an improved version of EZW, called SPITH (Set Partitioning in Hierarchical Trees). This method manages the subdivision of the trees with better technique and achieves better results than EZW by means of compression ratio and image quality. The SPITH algorithm groups the wavelet coefficients in order to store the significant information, even without taking into account the final arithmetic encoding stage in EZW encoder. In the other subsequent work a joint spacefrequency quantization scheme was proposed [22]. In this approach, the images are modeled by a linear combination of compacted energy in both frequency and spatial domains. In the other method called Group Testing for Wavelets (GTW), the wavelet coefficients are divided into different classes in a bit plane and each class are coded with a different group tester [23]. In GTW method, it is considered that, each class of coefficients has a different context and each group tester is a general entropy coder. Ratedistortion performances show that the GTW method is significantly better than SPITH method and close to SPITH-AC (with arithmetic coding). A new wavelet-transformation algorithm called The JPEG2000 was released by an ISO standardization committee in January 2001. The new algorithm was offering improved image quality at very high compression ratios [24].

Principal Component Analysis (PCA), or equivalently called Karhunen-Loève Transform has been widely used as an efficient method to provide an informative and low dimensional representation of the data from which important features can be extracted $[25,26]$. The method provides an optimal transform in order to decorrelate the data in the least mean square (LMS) sense among all linear orthogonal transforms. PCA is a linear orthogonal transform from an $m$-dimensional space to $p$-dimensional space, $p \leq m$, so that the coordinates of the original data in the new space are uncorrelated and the greatest amount of the variance of the original data is kept by only a few coordinates. The principal components can be obtained by solving an eigenvalue problem of the covariance or correlation matrix. The first $p$ eigenvectors correspond to $p$ principal components and span the principal subspace of dimension $p$. The Eigenvectors and associated eigenvalues are extracted by very well-known numerical algorithms [27]. In PCA, computation of the covariance matrix is not practical for handling high-dimensional data. In order to 
reduce the computational complexity of the PCA, several online neural network approaches were proposed. In Oja's algorithm the first or equivalently the most important and the last eigenvectors were extracted [26]. Generalized Hebbian Algorithm (GHA) extracts not only these two eigencomponents but also all the other eigencomponents [28]. In order to improve the convergence rate or speeding up the algorithm, an improved version of the GHA called adaptive principal component extraction was proposed [29]. The successive application of modified Hebbian learning algorithm was proposed as an extension of the GHA [30]. In the subsequent works the eigencomponents were recursively extracted $[31,32]$. The cascade recursive least square PCA algorithm (CRLS-PCA) was proposed in order to resolve the accumulation of errors in the extraction of large number of eigencomponents $[33,34]$. It is shown that the CRLS-PCA algorithm outperforms other neural network-based PCA approaches [35].

It well known that the PCA is a data-dependent transform. In other words, as the transform matrix is built based on the covariance matrix for a particular input image, it is possible to lose the approximation ability when the input image data is changed. In order to resolve this problem, improved versions of the PCA method have been proposed. It should be noted that among all these methods only very few of them take into account the PCA as a universal or semiuniversal image encoder. In recent works, image compression performance of the plain PCA is improved by proposed nonlinear and flexible PCA frameworks [36].

More recently, a variety of powerful and sophisticated DCT- [37-39] and Wavelet- [40-42] and PCA- [43-46] based compression schemes have been developed and established. Comparative results on these methods show that the compression performance of DCT based coders (JPEG) generally degrades the image especially at low bit rates mainly because of underlying block-based DCT scheme. Waveletbased coding methods provide considerable improvements in image quality at higher compression ratios [47]. On the other hand, software or hardware implementation of the DCT is less expensive than that of the wavelet transform [48]. PCA or Karhunen-Loève Transform (KLT) has computational complexity based on the computation of the covariance matrix of the training data. Despite being able to achieve much faster compression than KLT, DCT leads to relatively great degradation of compression quality at the same compression ratio compared to KLT [49].

In our previous works, $[50,51]$, a novel method referred to as SYMPES (systematic procedure for predefined envelope and signature sequences) was introduced and implemented on the representation of the $1 \mathrm{D}$ signals such as speech signals. The performance analysis and the comparative results of the SYMPES with respect to the other conventional speech compression algorithms were also presented in the other work [50]. The structure of the SYMPES is based on the creation of the so-called predefined signature and envelope sets which are speaker and language independent. The method is also implemented in the compression of the biosignals such as ECG [52] and EEG [53] signals.
In this paper, a new block-based image compression scheme is proposed based on generation of fixed block sets called Classified Energy Blocks (CEBs) and Classified Pattern Blocks (CPBs). All these unique block sets are associated under the framework called Classified Energy and Pattern Blocks (CEPBs). Basically, the method contains three main stages: (1) generation of the CEPB, (2) encoding process which contains construction of the energy and pattern building blocks of the image to be reconstructed and obtaining the encoding parameters, and (3) decoding (reconstruction) process of the input image using the encoding parameters from the already located CEPB in the receiver part (decoding).

In this paper, the performance of the method is measured on the experiments carried out in two groups. In the first group of experiments, the size of the image block vectors ( $L_{\mathrm{IBV}}$ ) is set to $L_{\mathrm{IBV}}=8 \times 8=64$ and three random orderings (threefold) of the training image data set are determined to construct three versions of the CEPB. Thus, the biasing effect in the evaluation stage is removed and then the average performances of the three CEPBs on the test data set (TDS) are reported. In the second group of experiments, in order to achieve higher compression ratios, all the images in the training image data set (excluding the images in the test data set) are used to construct the CEPB with $L_{\mathrm{IBV}}=16 \times$ $16=256$. It is observed that, when the compression ratio reaches the higher levels, degradation in the image caused by the blocking effect is getting visible. But, it is also worth to mention that, the image quality is at $27 \mathrm{~dB}$ level on the average even at 85,33:1 compression ratio.

In this paper, in order to remove the blocking effect and improve the PSNR levels, a postprocessing filter is used on the reconstructed images and the PSNR levels are improved in the range of $0.5-1 \mathrm{~dB}$. The speed of the algorithm and the compression ratio are also increased by adjusting the size of the CEPB with an efficient clustering algorithm in both group of experiments.

The preliminary results [54] and the results in this paper are obtained with new experimental setup and additional processes (3-Fold evaluation, clustering and postfiltering) the proposed method promises high compression ratio and acceptable image quality in terms of PSNR levels even at low bit rates.

\section{Method}

The method proposed consists of three major parts: construction of the classified energy and pattern blocks (CEPBs), construction of the energy and pattern blocks of the input image to be reconstructed and obtaining the encoding parameters (encoding process) and reconstruction (decoding) process using the mathematical model proposed.

Construction of the Classified Energy and Pattern Blocks $(C E P B)$. In this stage, we choose very limited number of image samples (training set) from the whole image set (image database) to construct the CEPB. In order to do this, we obtain energy and pattern blocks of each image files in the training set and then concatenate energy blocks 


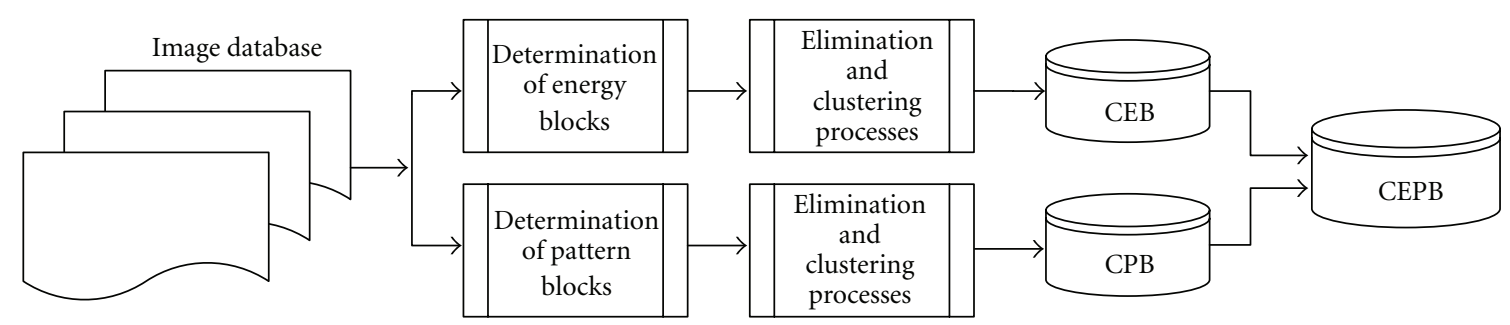

FIgURE 1: Construction process of the CEPB.

and pattern blocks separately. After an elimination process which eliminates the similar energy and pattern blocks in their classes, a classified (or unique) CEPB are obtained as illustrated in Figure 1.

Construction of the Energy and Pattern Blocks of the Input Image to Be Reconstructed and Obtaining the Encoding Parameters (Encoding Process). In this part, the energy and pattern blocks are constructed using the same process applied in the construction of the CEPB excluding the main elimination part. In this process, energy and pattern blocks of the input image are compared to the blocks located in the CEPB using a matching algorithm and encoding parameters are determined. The encoding parameters for each block are the optimum scaling coefficient and the index numbers of best representative classified energy and pattern blocks in the CEPB which matches the energy and pattern blocks of the input image to be reconstructed, respectively. The scheme of the encoding process is shown in Figure 2.

Reconstruction (Decoding) Process. This part includes the image reconstruction (or decoding) process. The input images (or test images) are reconstructed block by block using the best representative parameters which are called block scaling coefficient (BSC), classified energy block index (IE) and classified pattern block index (IP) based on the mathematical model as presented in the following section. The scheme of the decoding process is presented in Figure 3.

In following subsections, we first present the details of our CEPB construction method which is exploited to reconstruct the input images. Then, we explain the construction of the energy and pattern blocks of the input image and how we employ the CEPB in the transmitter part to obtain the encoding parameters of the input image. Finally, we briefly describe the reconstruction (decoding) process using the encoding parameters which are sent from the transmitter and reconstruction of the input image block by block using these parameters employing the CEPB which is already located in the receiver part.

2.1. Construction of the Classified Energy and Pattern Blocks (CEPBs). Let the image data $\operatorname{Im}(m, n)$ be an $M \times N$ (in our cases, $M=N=512$ ) matrix with integer entries in the range of 0 to 255 or the real values in the range of 0 to 1 where $m$ and $n$ are row and column pixel indices of the whole image, respectively. The input image is first divided into nonoverlapping image blocks, $B_{r, c}$ of size $i \times j$, where the image block size is $i=j=8,16$, and so forth. The pixel location of the $k$ th row and $l$ th column of the block, $B_{r, c}$ is represented by $P_{B_{r, c}, k, l}$, where the pixel indices are $k=1$ to $i$ and $l=1$ to $j$. In this case, the total number of blocks in the $\operatorname{Im}(m, n)$ will be equal to $N_{B}=(M \times N) /(i \times j)$. The indices $r$ and $c$ of the $B_{r, c}$ are in the range of 1 to $M / i$ and $N / j$, respectively. As illustrated in Figure 4, in our method, all the image blocks $B_{r, c}$ from left to the right direction are reshaped as column vectors and constructed a new matrix denoted as $B_{\mathrm{Im}}$.

In the construction of the two block sets (CEPBs), a certain number of image files are determined as a training set from the whole image database. Each image file in the training set is divided into the $8 \times 8(i=j=8)$ or $16 \times$ $16(i=j=16)$ image blocks, and then each image block is reshaped as a column vector called image block vector (vector representation of the image block) which has $i \times j$ pixels.

All the image files have the same number of pixels $(512 \times$ $512=262,144)$ and equal number of image blocks $N_{B}$. After the blocking process the image matrix can be written as follows:

$\mathrm{Im}$

$$
=\left[\begin{array}{ccccc}
B_{1,1} & B_{1,2} & \cdots & B_{1,(N / j)-1} & B_{1,(N / j)} \\
B_{2,1} & B_{2,2} & \cdots & B_{2,(N / j)-1} & B_{2,(N / j)} \\
\cdots & \cdots & \cdots & \cdots & \cdots \\
B_{(M / i)-1,1} & B_{(M / i)-1,2} & \cdots & B_{(M / i)-1,(N / j)-1} & B_{(M / i)-1,(N / j)} \\
B_{(M / i), 1} & B_{(M / i), 2} & \cdots & B_{(M / i),(N / j)-1} & B_{(M / i),(N / j)}
\end{array}\right] .
$$

The matrix Im is transformed to a new matrix, $B_{\operatorname{Im}}$, which its column vectors are the image blocks of the matrix, Im

$$
B_{\operatorname{Im}}=\left[\begin{array}{lllllll}
B_{1,1} & \cdots & B_{1,(N / j)} & B_{2,1} & \cdots & \cdots & B_{(M / i),(N / j)}
\end{array}\right] .
$$

The columns of the matrix $B_{\mathrm{Im}}$ are called image block vector (IBV) and the length of the IBV is represented by $L_{\mathrm{IBV}}=i \times j(8 \times 8=64$ or $16 \times 16=256$, etc. $)$.

As it is explained above, in the method that we proposed the IBVs of an image can be represented by a mathematical model which consists of the multiplication of the three quantities; scaling factor, classified pattern and energy blocks. 


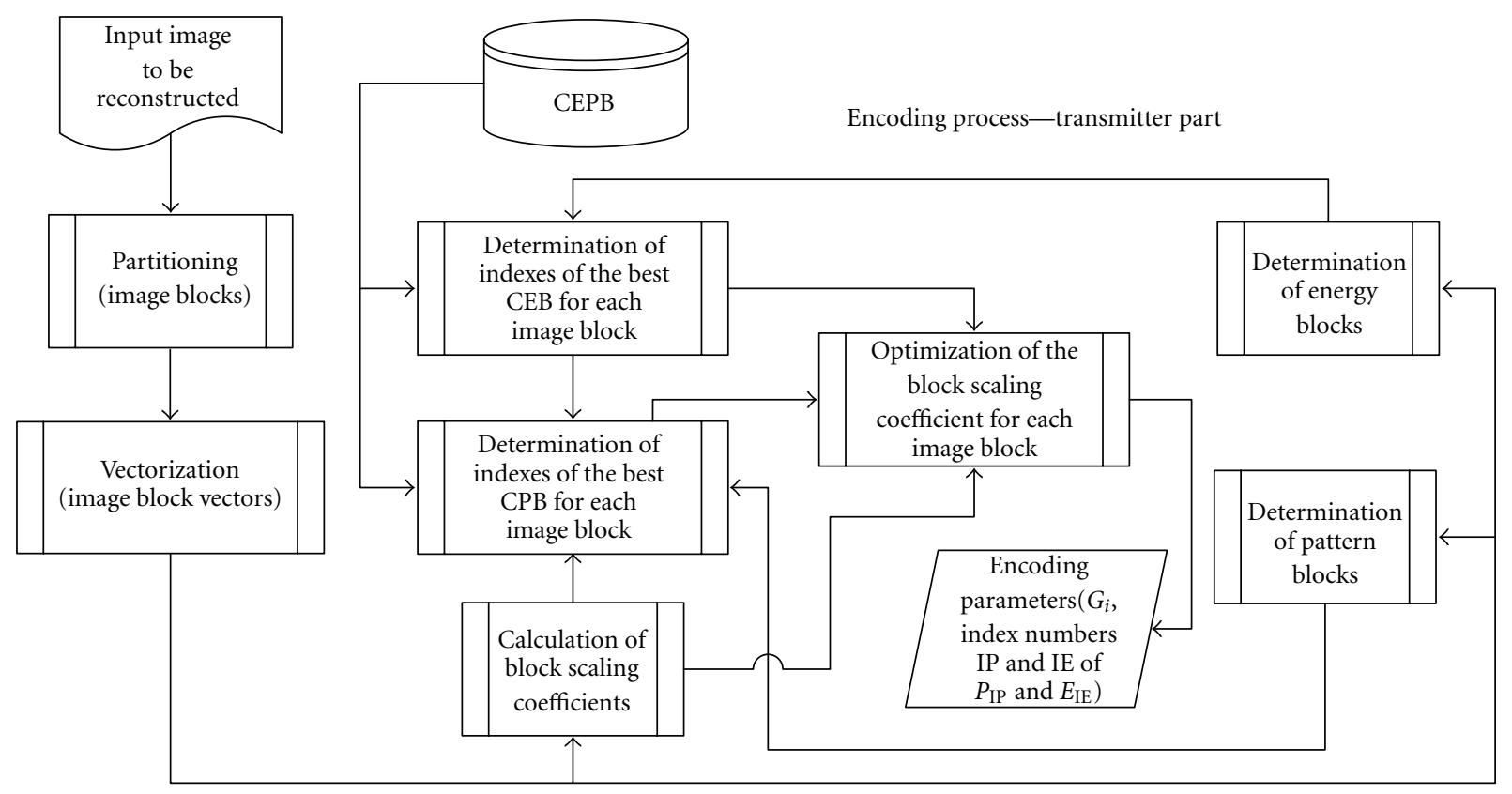

FIGURE 2: Encoding process.

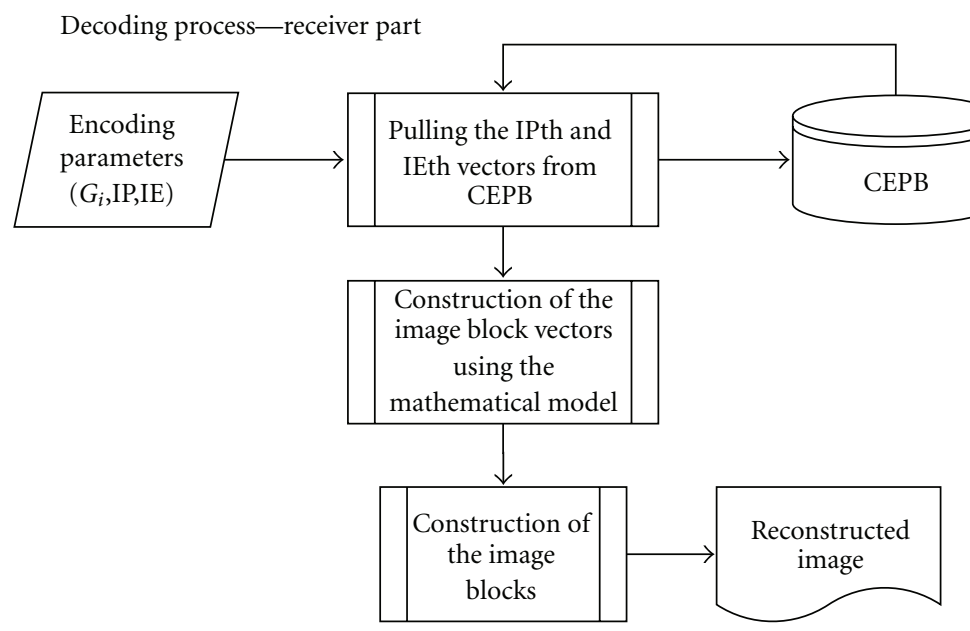

FIGURE 3: Decoding process.

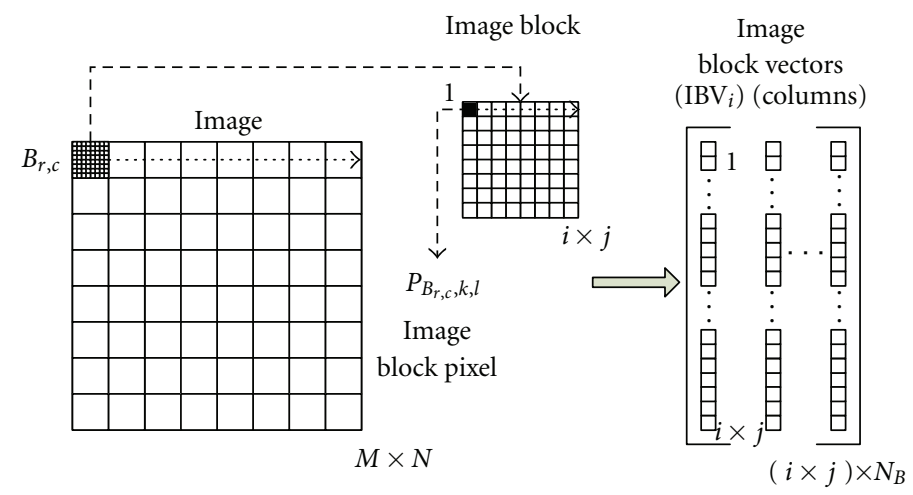

FIGURE 4: Partitioning of an image into the image blocks and reshaping as vector form. 
In our method it is proposed that any $i$ th IBV of length $L_{\mathrm{IBV}}$ can be approximated as $\mathrm{IBV}_{i}=G_{i} P_{\mathrm{IP}} E_{\mathrm{IE}},\left(i=1, \ldots, N_{B}\right)$ where the scaling coefficient, $G_{i}$ of the IBV is a real constant, IP $\in\left\{1,2, \ldots, N_{\text {IP }}\right\}$, IE $\in\left\{1,2, \ldots, N_{\text {IE }}\right\}$ are the index number of the CPB and index number of the CEB, where $N_{\text {IP }}$ and $N_{\mathrm{IE}}$ are the total number of the $\mathrm{CPB}$ and CEB indices, respectively. IP, IE, $N_{\mathrm{IP}}$, and $N_{\mathrm{IE}}$ are all integers.

The CEB in the vector form is represented as $E_{\mathrm{IE}}^{T}=$ $\left[\begin{array}{llll}e_{\mathrm{IE} 1} & e_{\mathrm{IE} 2} & \cdots & e_{\mathrm{IEL}} \mathrm{IBV}\end{array}\right]$ and it is generated utilizing the luminance information of the images and it contains basically the energy characteristics of $\mathrm{IBV}_{i}$ under consideration in broad sense. Furthermore, it will be shown that the quantity $G_{i} E_{\mathrm{IE}}$ carries almost maximum energy of $\mathrm{IBV}_{i}$ in the least mean square (LMS) sense. In this multiplication expression the contribution of the $G_{i}$ is to scale the luminance level of the $\operatorname{IBV}_{i}$.

$P_{\mathrm{IP}}$ is $\left(L_{\mathrm{IBV}} \times L_{\mathrm{IBV}}\right)$ diagonal matrix such that

$$
P_{\mathrm{IP}}=\operatorname{diag}\left[\begin{array}{lllll}
p_{\mathrm{IP} 1} & p_{\mathrm{IP} 2} & p_{\mathrm{IP} 3} & \cdots & p_{\mathrm{IP} L_{\mathrm{IBV}}}
\end{array}\right],
$$

$P_{\mathrm{IP}}$ acts as a pattern term on the quantity $G_{i} E_{\mathrm{IE}}$ which also reflects the distinctive properties of the image block data under consideration.

It is well known that each IBV can be spanned in a vector space formed by the orthonormal vectors $\left\{\phi_{i k}\right\}$. Let the real orthonormal vectors be the columns of a transposed transformation matrix $\left(\Phi_{i}^{T}\right)$

$$
\Phi_{i}^{T}=\left[\begin{array}{llll}
\phi_{i 1} & \phi_{i 2} & \cdots & \phi_{i L_{\mathrm{IBV}}}
\end{array}\right]
$$

It is evident that

$$
\mathrm{IBV}_{i}=\Phi_{i}^{T} G_{i}
$$

where

$$
G_{i}^{T}=\left[\begin{array}{llll}
g_{1} & g_{2} & \cdots & g_{L_{\mathrm{IBV}}}
\end{array}\right]
$$

From the property of $\Phi_{i}^{T}=\Phi_{i}^{-1}$, the equations $\Phi_{i} \mathrm{IBV}_{i}=$ $\Phi_{i} \Phi_{i}^{-1} G_{i}$ and $G_{i}=\Phi_{i} \mathrm{IBV}_{i}$ can be obtained, respectively.

Thus, $\mathrm{IBV}_{i}$ can be written as a weighted sum of these orthonormal vectors

$$
\mathrm{IBV}_{i}=\sum_{k=1}^{L_{\mathrm{IBV}}} g_{k} \phi_{i k}, \quad k=1,2,3, \ldots, L_{\mathrm{IBV}}
$$

From the above equation, the coefficients of the IBVs can be obtained as

$$
g_{k}=\phi_{i k}^{T} \mathrm{IBV}_{i}, \quad k=1,2,3, \ldots, L_{\mathrm{IBV}} .
$$

Let $\mathrm{IBV}_{i t}=\sum_{k=1}^{t} g_{k} \phi_{i k}$ be the truncated version of $\mathrm{IBV}_{i}$ such that $1 \leq t \leq L_{\mathrm{IBV}}$. It is noted that if $t=L_{\mathrm{IBV}}$, then $\mathrm{IBV}_{i}$ will be equal to $\mathrm{IBV}_{i t}$. In this case, the approximation error $\left(\varepsilon_{t}\right)$ is given by

$$
\varepsilon_{t}=\mathrm{IBV}_{i}-\mathrm{IBV}_{i t}=\sum_{k=t+1}^{L_{\mathrm{IBV}}} g_{k} \phi_{i k} .
$$

In this equation, $\phi_{i k}$ are determined by minimizing the expected value of the error vector with respect to $\phi_{i k}$ in the LMS sense. The above-mentioned LMS process results in the following eigenvalue problem [55]. Eventually $\phi_{i k}$ are computed as the eigenvectors of the correlation matrix $\left(R_{i}\right)$ of the $\mathrm{IBV}_{i}$. By using orthonormality condition, the LMS error is given by

$$
\varepsilon_{t} \varepsilon_{t}^{T}=\sum_{k=t+1}^{L_{\mathrm{IBV}}} g_{k}^{2}
$$

Let $J_{t}$ designate the expected value of the total squared error $\varepsilon_{t} \varepsilon_{t}^{T}$. Then,

$$
J_{t}=\mathrm{E}\left[\varepsilon_{t} \varepsilon_{t}^{T}\right]=\sum_{k=t+1}^{L_{\mathrm{IBV}}} \mathrm{E}\left[g_{k}^{2}\right],
$$

$$
\mathrm{E}\left[g_{k}^{2}\right]=\mathrm{E}\left[\phi_{i k}^{T}\left(\operatorname{IBV}_{i}^{T} \operatorname{IBV}_{i}\right) \phi_{i k}\right]=\phi_{i k}^{T} R_{i} \phi_{i k},
$$

where $R_{i}=\mathrm{E}\left[\mathrm{IBV}_{i}^{T} \mathrm{IBV}_{i}\right]$ is defined as the correlation matrix of $\mathrm{IBV}_{i}$. In order to obtain the optimum transform, it is desired to find $\phi_{i k}$ that minimizes $J_{t}$ for a given $t$, subject to the orthonormality constraint. Using Lagrange multipliers $\lambda_{k}$, we minimize $J_{t}$ by taking the gradient of the equation obtained above with respect to $\phi_{i k}$ :

$$
\begin{gathered}
J_{t}=\sum_{k=t+1}^{L_{\mathrm{IBV}}}\left[\phi_{i k}^{T} R_{i} \phi_{i k}-\lambda_{k}\left(\phi_{i k}^{T} \phi_{i k}-1\right)\right] \\
\frac{\partial J_{t}}{\partial \phi_{i k}}=\frac{\partial}{\partial \phi_{i k}}\left[\sum_{k=t+1}^{L_{\mathrm{IBV}}}\left[\phi_{i k}^{T} R_{i} \phi_{i k}-\lambda_{k}\left(\phi_{i k}^{T} \phi_{i k}-1\right)\right]\right]=0 \\
2 R_{i} \phi_{i k}-2 \lambda_{k} \phi_{i k}=0 \\
R_{i} \phi_{i k}=\lambda_{k} \phi_{i k}
\end{gathered}
$$

$R_{i}$ is the correlation matrix. It is real, symmetric with respect to its diagonal elements, positive semidefinite, and Toeplitz matrix [56]:

$R_{i}$

$$
\begin{aligned}
& =\left[\begin{array}{ccccc}
r_{i}(1) & r_{i}(2) & r_{i}(3) & \cdots & r_{i}\left(L_{\mathrm{IBV}}\right) \\
r_{i}(2) & r_{i}(1) & r_{i}(2) & \cdots & r_{i}\left(L_{\mathrm{IBV}}-1\right) \\
r_{i}(3) & r_{i}(2) & r_{i}(1) & \cdots & r_{i}\left(L_{\mathrm{IBV}}-2\right) \\
\vdots & \vdots & \vdots & \ddots & \vdots \\
r_{i}\left(L_{\mathrm{IBV}}\right) & r_{i}\left(L_{\mathrm{IBV}}-1\right) & r_{i}\left(L_{\mathrm{IBV}}-2\right) & \cdots & r_{i}(1)
\end{array}\right], \\
& r_{i}(d+1) \\
= & \frac{1}{L_{\mathrm{IBV}}} \sum_{j=\left[(i-1) \cdot L_{\mathrm{IBV}}+1\right]}^{\left[\left(i L_{\mathrm{IBV}}\right)-d\right]} x_{j} x_{j+d}, \quad d=0,1,2, \ldots, \mathrm{L}_{\mathrm{IBV}}-1 .
\end{aligned}
$$

Obviously, $\lambda_{i k}$ and $\phi_{i k}$ are the eigenvalues and eigenvectors of the eigenvalue problem under consideration. It is well 
known that the eigenvalues of $R_{i}$ are also real, distinct, and nonnegative. Moreover, the eigenvectors $\phi_{i k}$ of the $R_{i}$ are all orthonormal. Let eigenvalues be sorted in descending order such that $\left(\lambda_{1 i} \geq \lambda_{2 i} \geq \lambda_{3 i} \geq \cdots \geq \lambda_{L_{\mathrm{IBV}}}\right)$ with corresponding eigenvectors. The total energy of the $\mathrm{IBV}_{i}$ is then given by $\mathrm{IBV}_{i}^{T} \mathrm{IBV}_{i}$ :

$$
\mathrm{IBV}_{i}^{T} \mathrm{IBV}_{i}=\sum_{k=1}^{L_{\mathrm{IBV}}} g_{i k}^{2}=\sum_{k=1}^{L_{\mathrm{IBV}}} \lambda_{i k} .
$$

Equation (15) may be truncated by taking the first $p$ principal components, which have the highest energy of the $\mathrm{IBV}_{i}$ such that

$$
\mathrm{IBV}_{i} \cong \sum_{k=1}^{p} g_{k} \phi_{i k} .
$$

The simplest form of (16) can be obtained by setting $p=$ 1. The eigenvector $\phi_{i k}$ is called energy vector. That is to say, the energy vector, which has the highest energy in the LMS sense, may approximate each image block belonging to the $\mathrm{IBV}_{i}$. Thus,

$$
\mathrm{IBV}_{i} \cong g_{1} \phi_{i 1}
$$

In this case, one can vary the $L_{\mathrm{IBV}}$ as a parameter in such way that almost all the energy is captured within the first term of (16) and the rest becomes negligible. That is why $\phi_{i 1}$ is called the energy vector since it contains most of the useful information of the original IBV under consideration. Once (17) is obtained, it can be converted to an equality by means of a pattern term $P_{i}$ which is a diagonal matrix for each IBV. Thus, $\mathrm{IBV}_{i}$ is computed as

$$
\mathrm{IBV}_{i}=G_{i} P_{i} \phi_{i 1}
$$

In (18), diagonal entries $p_{i r}$ of the matrix $P_{i}$ are determined in terms of the entries of $\phi_{i 1 r}$ of the energy vector $\phi_{i 1}$ and the entries (pixels) $\mathrm{IBV}_{i r}$ of the $\mathrm{IBV}_{i}$ by simple division. Hence,

$$
p_{i r}=\frac{\mathrm{IBV}_{i r}}{G_{i} \phi_{i 1 r}}, \quad\left(r=1,2, \ldots, L_{\mathrm{IBV}}\right) .
$$

In essence, the quantities $p_{i r}$ of (19) somewhat absorb the energy of the terms eliminated by truncation of (16).

In this paper, several tens of thousands of IBVs were investigated and several thousands of energy and pattern blocks were generated. It was observed that the energy and the pattern blocks exhibit repetitive similarities. In this case, one can eliminate the similar energy and pattern blocks and thus, constitute the so-called classified energy and classified pattern block sets with one of a kind or unique blocks. For the elimination process Pearsons correlation coefficient (PCC) [57] is utilized. PCC is designated by $\rho_{Y Z}$ and given as

$$
\begin{aligned}
& \rho_{Y Z} \\
& \sqrt{\left[\sum_{i=1}^{L} y_{i}^{2}-\left(\sum_{i=1}^{L} y_{i}\right)^{2} / L\right] \cdot\left[\sum_{i=1}^{L} z_{i}^{2}-\left(\sum_{i=1}^{L} z_{i}\right)^{2} / L\right]}
\end{aligned}
$$

In (20) $Y=\left[\begin{array}{llll}y_{1} & y_{2} & \cdots & y_{L}\end{array}\right]$ and $Z=\left[\begin{array}{llll}z_{1} & z_{2} & \cdots & z_{L}\end{array}\right]$ are two sequences subject to comparison, where $L$ is the length of the sequences. It is assumed that the two sequences are almost identical if $0.9 \leq \rho_{Y Z} \leq 1$. Hence, similar energy and pattern blocks are eliminated accordingly.

During the execution of the elimination stage, it is observed that similarity rate of the energy blocks are much higher than the pattern blocks. Because of huge differences in the similarity rate or in other words elimination rate, the numbers of classified energy blocks in the CEPB are very limited. This is natural because energy blocks reflect the luminance information of the image blocks, while pattern blocks carry the pattern or variable information in the image blocks. This is in reality related to tasks of these blocks in the method as explained in the beginning of this section.

For the elimination, PCC is set to $\rho_{Y Z}=0,98$ which is very close to $\rho_{Y Z}=1$ but it can be relaxed (or adjusted) according to the desired number (size) of classified energy and pattern blocks in the CEPB.

In the elimination stage, first the similar energy and pattern block groups are constructed and one representative energy and one representative pattern block are determined for each group by averaging all the blocks in the groups. These representative energy and pattern blocks are renamed as classified energy and pattern blocks and constitute the CEPB.

Thus, the energy blocks which have unique shapes are combined under the set called classified energy block $\mathrm{CEB}=$ $\left\{\mathrm{E}_{n_{i e}} ; n_{i e}=1,2,3, \ldots, N_{\mathrm{IE}}\right\}$ set. The integer $N_{\mathrm{IE}}$ designates the total number of elements in this set. Similarly, reduced pattern blocks are combined under the set called classified pattern block $\mathrm{CPB}=\left\{P_{n_{i p}} ; n_{i p}=1,2,3, \ldots, N_{\mathrm{IP}}\right\}$ set. The $N_{\text {IP }}$ designates the total number of unique pattern sequences in $\mathrm{CPB}$ set. Some similar energy and pattern blocks are depicted in Figures 5 and 6, respectively.

Computational steps and the details of the encoding and decoding algorithms are given in Sections 2.2 and 2.3, respectively.

\subsection{Encoding Algorithm}

Inputs. The inputs include the following:

(1) image file $\{\operatorname{Im}(m, n), M \times N=512 \times 512\}$ to be encoded;

(2) size of the IBV of the $\operatorname{Im}(m, n)\left(L_{\mathrm{IBV}}=i \times j=8 \times 8=\right.$ 64 or $\left.L_{\mathrm{IBV}}=i \times j=16 \times 16=256\right)$;

(3) the $\mathrm{CEPB}\left(\mathrm{CEB}=\left\{E_{\mathrm{IE}} ; \mathrm{IE}=1,2, \ldots, N_{\mathrm{IE}}\right\}\right.$ and $\mathrm{CPB}=$ $\left.\left\{P_{\mathrm{IP}} ; \mathrm{IP}=1,2, \ldots, N_{\mathrm{IP}}\right\}\right)$ located in the transmitter part.

\section{Computational Steps.}

Step 1. Divide $\operatorname{Im}(m, n)$ into the image blocks, and then construct the $B_{\mathrm{Im}}$.

Substep 2.1. For each $\mathrm{IBV}_{i}$ pull an appropriate $E_{\mathrm{IE}}$ from CEB such that the distance or the total error $\delta_{\mathrm{IE}}=$ $\left\|\mathrm{IBV}_{i}-G_{\mathrm{IE}} E_{\mathrm{IE}}\right\|^{2}$ is minimum for all $\mathrm{I} \tilde{\mathrm{E}}=1,2,3, \ldots$, IE, $\ldots, N_{\mathrm{IE}}$. This step yields the index $I E$ of the $E_{\mathrm{IE}}$. In this case, $\delta_{\mathrm{IE}}=\min \left\{\left\|\mathrm{IBV}_{i}-G_{\mathrm{IE}} E_{\mathrm{IE}}\right\|^{2}\right\}=\left\|\mathrm{IBV}_{i}-G_{\mathrm{IE}} E_{\mathrm{IE}}\right\|^{2}$. 


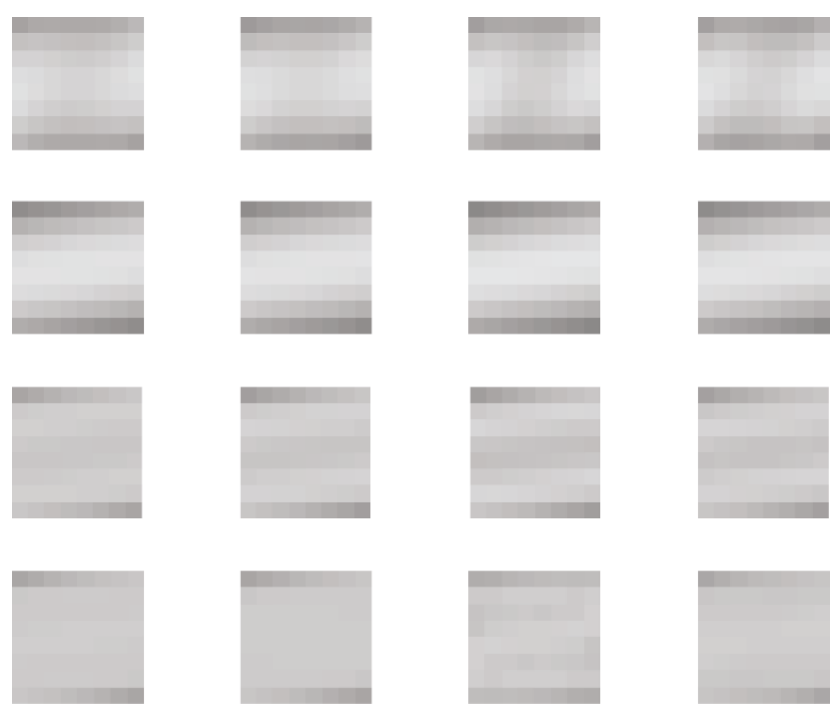

Figure 5: Some of the similar energy blocks ( 4 similar energy blocks from left to right in each set).

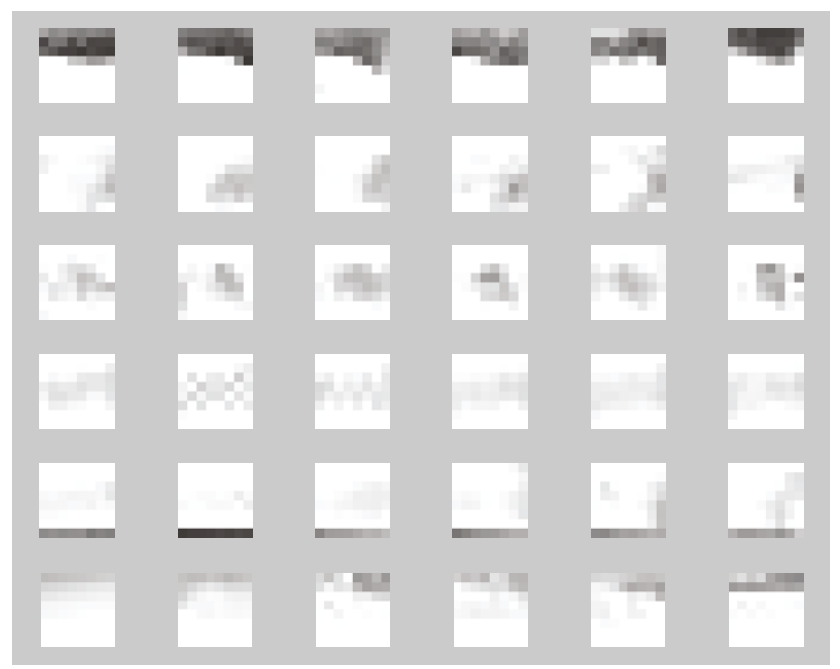

FIGURE 6: Some of the similar pattern blocks (6 similar pattern blocks from left to right in each set).

Substep 2.2. Store the index number $I E$ that refers to $E_{\mathrm{IE}}$, in this case, $\mathrm{IBV}_{i} \approx G_{\mathrm{IE}} E_{\mathrm{IE}}$.

Substep 3.3. Pull an appropriate $P_{\mathrm{IP}}$ from $\mathrm{CPB}$ such that the error is further minimized for all I $\widetilde{P}=1,2,3, \ldots, I P, \ldots, N_{\text {IP }}$. This step yields the index $I P$ of $P_{\text {IP }}$

$$
\delta_{\mathrm{IP}}=\min \left\{\left\|\mathrm{IBV}_{i}-G_{\mathrm{IE}} P_{\mathrm{IP}} E_{\mathrm{IE}}\right\|^{2}\right\}=\left\|\mathrm{IBV}_{i}-G_{\mathrm{IE}} P_{\mathrm{IP}} E_{\mathrm{IE}}\right\|^{2} .
$$

Substep 3.4. Store the index number IP that refers to $P_{\mathrm{IP}}$. At the end of this step, the best $E_{\mathrm{IE}}$ and the best $P_{\mathrm{IP}}$ are found by appropriate selections. Hence, the $\mathrm{IBV}_{i}$ is best described in terms of the patterns of $P_{\mathrm{IP}}$ and $E_{\mathrm{IE}}$, that is, $\mathrm{IBV}_{i} \cong G_{\mathrm{IE}} P_{\mathrm{IP}} E_{\mathrm{IE}}$.
Step 4. Having fixed $P_{\mathrm{IP}}$ and $E_{\mathrm{IE}}$, one can replace $G_{\mathrm{IE}}$ by computing a new block scaling coefficient $G_{i}=$ $\left(P_{\mathrm{IP}} E_{\mathrm{IE}}\right)^{T} \mathrm{IBV}_{i} /\left(P_{\mathrm{IP}} E_{\mathrm{IE}}\right)^{T}\left(P_{\mathrm{IP}} E_{\mathrm{IE}}\right)$ to further minimize the distance between the vectors $\mathrm{IBV}_{i}$ and $G_{\mathrm{IE}} P_{\mathrm{IP}} E_{\mathrm{IE}}$ in the LMS sense. In this case, the global minimum of the error is obtained and it is given by $\delta_{\mathrm{Global}}=\left\|\mathrm{IBV}_{i}-G_{i} P_{\mathrm{IP}} E_{\mathrm{IE}}\right\|^{2}$. At this step, $\mathrm{IBV}_{A i}=G_{i} P_{\mathrm{IP}} E_{\mathrm{IE}}$.

\subsection{Decoding Algorithm}

Inputs. The inputs include the following:

(1) the encoding parameters $G_{i}$, IP and IE which best represent the corresponding image block vector $\mathrm{IBV}_{i}$ of the input image (These parameters are received from the transmitter part for each image block vector of the input image);

(2) size of the $\mathrm{IBV}_{i}$ of the $\operatorname{Im}(m, n)\left(L_{\mathrm{IBV}}=i \times j=8 \times 8=\right.$ 64 or $\left.L_{\mathrm{IBV}}=i \times j=16 \times 16=256\right)$;

(3) the $\mathrm{CEPB}\left(\mathrm{CEB}=\left\{E_{\mathrm{IE}} ; \mathrm{IE}=1,2, \ldots, N_{\mathrm{IE}}\right\}\right.$ and $\mathrm{CPB}=$ $\left.\left\{P_{\mathrm{IP}} ; \mathrm{IP}=1,2, \ldots, N_{\mathrm{IP}}\right\}\right)$ located in the receiver part.

\section{Computational Steps.}

Step 1. After receiving the encoding parameters $G_{i}$, IP, and IE of the IBV $\mathrm{I}_{i}$ from the transmitter, the corresponding IEth classified energy and IPth classified pattern blocks are pulled from the CEPB.

Step 2. Approximated image block vector $\mathrm{IBV}_{A i}$ is constructed using the proposed mathematical model $\mathrm{IBV}_{A i}=$ $G_{i} P_{\mathrm{IP}} E_{\mathrm{IE}}$.

Step 3. The previous steps are repeated for each IBV to generate approximated version $\left(\widehat{B}_{\mathrm{Im}}\right)$ of the $B_{\mathrm{Im}}$

$$
\widehat{B}_{\operatorname{Im}}=\left[\begin{array}{lllllll}
\hat{B}_{1,1} & \cdots & \widehat{B}_{1,(N / j)} & \hat{B}_{2,1} & \cdots & \cdots & \hat{B}_{(M / i),(N / j)}
\end{array}\right] .
$$

Step 4. $\widehat{B}_{\mathrm{Im}}$ is reshaped to obtain the decoded (reconstructed) version of the original image data as follows:

$\widehat{\mathrm{Im}}$

$$
=\left[\begin{array}{ccccc}
\hat{B}_{1,1} & \hat{B}_{1,2} & \cdots & \widehat{B}_{1,(N / j)-1} & \widehat{B}_{1,(N / j)} \\
\hat{B}_{2,1} & \hat{B}_{2,2} & \cdots & \widehat{B}_{2,(N / j)-1} & \widehat{B}_{2,(N / j)} \\
\cdots & \cdots & \cdots & \cdots & \cdots \\
\hat{B}_{(M / i)-1,1} & \hat{B}_{(M / i)-1,2} & \cdots & \hat{B}_{(M / i)-1,(N / j)-1} & \hat{B}_{(M / i)-1,(N / j)} \\
\hat{B}_{(M / i), 1} & \hat{B}_{(M / i), 2} & \cdots & \widehat{B}_{(M / i),(N / j)-1} & \widehat{B}_{(M / i),(N / j)}
\end{array}\right] .
$$

2.4. Introducing the Blocking Effect and Postfiltering. It is well known for block-coded image compression schemes, the image is partitioned into blocks, and certain transform is performed on each individual block. In particular, at low bit rates, since each block is represented primarily by the 
first transform coefficient, the rectangular block structure becomes very visible because of the presentation of the discontinuity at block boundaries. There are several existing techniques that attempt to remove blocking effect or artifacts of the low bit-rate coded images.

In this frame-based work, the blocking effect occurs especially at low bit rates. Especially, when the size of the CEPB is highly reduced or the size of the image blocks $\left(L_{\mathrm{IBV}}\right)$ are increased from $8 \times 8$ to $16 \times 16$, the effect of the blocking becomes visible. In order to remove these effects a 2D Savitzky-Golay filtering [58] or smoothing process is applied after the reconstruction process at the receiver side. The aim of this postprocessing is smoothing the block boundaries so that both the PSNR and visual perception of the reconstructed image can be improved.

At the end of the reconstruction process for all the images in the first and second groups of experiments, the SavitzkyGolay filter is applied on the reconstructed images. The PSNR performances of the filter of various window sizes and different polynomial orders are compared by an iterative algorithm. After all these comparisons, it is observed that, for the first group of experiments, the frame size and the order of the polynomial which maximizes the PSNR level are found as 5 and 3 , respectively. The frame size and the order of the polynomial are determined as 7 and 3 for the second group of experiments. The PSNR and MSE performances are noticed before and after the filtering process and at the end of the evaluation process, it is seen that the PSNR level is increased about $0.5-1 \mathrm{~dB}$ compared to the results obtained without filtering process for the first and second group of experiments.

\section{Experiments and Results}

3.1. Data Sets. In our experiments, 67 gray-scale, 8 bits/pixel, $512 \times 512$ JPEG images [59] were used. The experiments were implemented in two groups. In the first group of experiments the size of the image blocks is $L_{\mathrm{IBV}}=i \times j=$ $8 \times 8=64$ while in the second $L_{\mathrm{IBV}}=i \times j=16 \times 16=256$.

In the first group of the experiments, three randomly selected file sets (Fold 1, Fold 2, and Fold 3) from the whole data set are used for training or construction of three different CEPBs. 12 image files which are randomly chosen from the rest of the data set are determined as the test data set (TDS). In the second group of experiments, we enlarged the training set to 55 files (TDA) excluding all the image files used in the test data set. All these cases are summarized in Table 1. The images in the training and test data sets are shown in Figures 7, 8, 9, and 10 for fold 1, fold 2, fold 3, and TDS, respectively.

3.2. Evaluation Metrics. Even though the HVS is the most reliable assessment tool to measure the quality of an image, the subjective quality measurement methods based on HVS such as mean opinion score (MOS) are not practical. Objective image and video quality metrics such as peak signal-to-noise ratio (PSNR) and mean squared error (MSE) are the most widely used objective image quality/distortion metrics and they can predict perceived image and video quality automatically. It should be also noted that these metrics are also criticized because they are not correlating well with the perceived quality measurement. Recently, image and video quality assessment research is trying to develop new objective image and video quality measures such as structural-similarity-based image quality assessment (SSIM) by considering HVS characteristics [60, 61]. Almost all the works in the literature consider the PSNR and MSE as an evaluation metrics to measure the quality of the image. Therefore, as a starting point at least for the comparisons, the performance of the newly proposed method is measured using PSNR and MSE metrics.

Peak Signal-to-Noise Ratio (PSNR). PSNR is the ratio between the signal's maximum power and the power of the signal's noise. The higher PSNR means better quality of the reconstructed image. The PSNR can be computed as

$$
\mathrm{PSNR}=20 \log _{10}\left(\frac{b}{\sqrt{\mathrm{MSE}}}\right),
$$

where $b$ is the largest possible value of the image signal (typically 255 or 1). The PSNR is given in decibel units (dB).

Mean Squared Error (MSE). MSE represents the cumulative squared error between the original and the reconstructed image, whereas PSNR represents a measure of the peak error. The MSE can be described as the mean of the square of the differences in the pixel values between the corresponding pixels of the two images. MSE can be written as

$$
\operatorname{MSE}=\frac{1}{M N} \sum_{i=1}^{M} \sum_{j=1}^{N}[\operatorname{Im}(m, n)-\widehat{\operatorname{Im}}(m, n)]^{2},
$$

where $\operatorname{Im}(m, n)$ and $\hat{\operatorname{Im}}(m, n)$ are the original and the reconstructed images, respectively. $M \times N$ is the dimension of the images. In our experiments the dimension of the images is $512 \times 512$.

Compression Ratio (CR). CR is defined as the ratio of the total number of bits required to represent the original and reconstructed image blocks. Other representation of the CR is the bpp:

$$
\mathrm{CR}=\frac{\text { bit }_{\text {original }}}{\text { bit }_{\text {reconstructed }}}, \quad \text { bpp }(\text { bit per pixel })=\frac{\sqrt{L_{\mathrm{IBV}}}}{\mathrm{CR}} .
$$

3.3. Experimental Results. In the first group of experiments the total number of bits required to represent the $8 \times 8$ blocks for each original image file is $(8 \times 8) \times 8$ bits $=512$ bits. In the first group of experiments the size of the CEPB is determined and fixed for all folds (3 Folds) by adjusting the PCC. Thus, total numbers of classified energy and pattern blocks are determined in the range of $2^{5}$ and $2^{14}$ in the CEB and CPB sets, respectively. It is also concluded that $\mathrm{N}_{\mathrm{IE}}$ and $\mathrm{N}_{\mathrm{IP}}$ are represented by 5 bits and 14 bits, respectively. For representation of the block scaling coefficient (BSC) 5 bits are good enough. As a result, 24 bits are required in total in order 

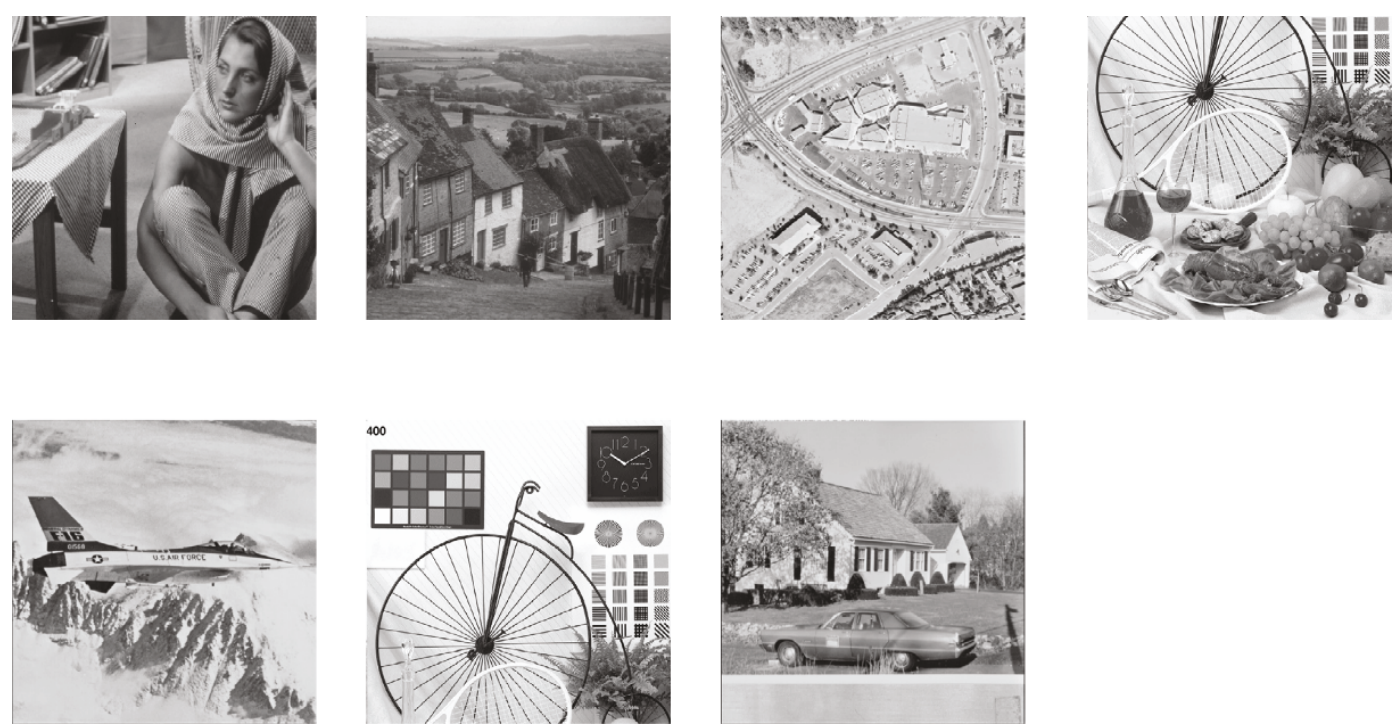

Figure 7: Image files in the training data set (Fold 1).
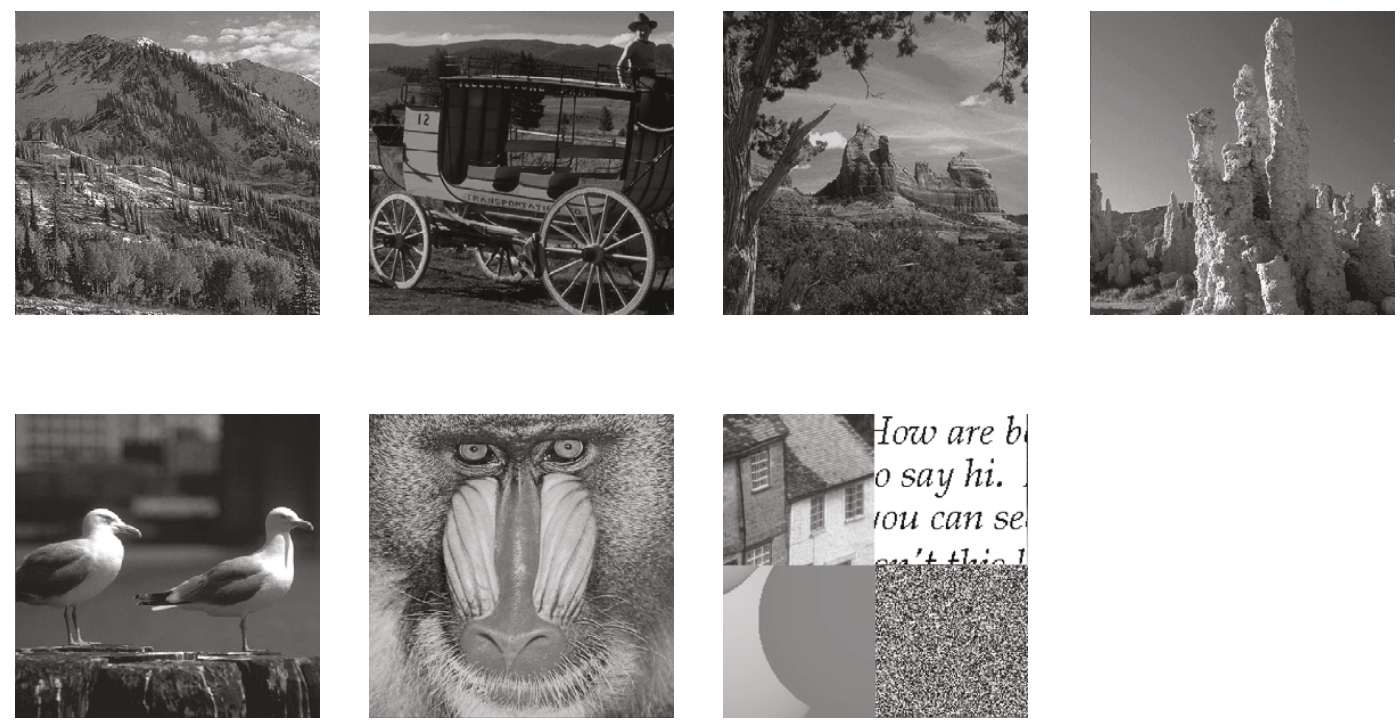

FIgURE 8: Image files in the training data set (Fold 2).
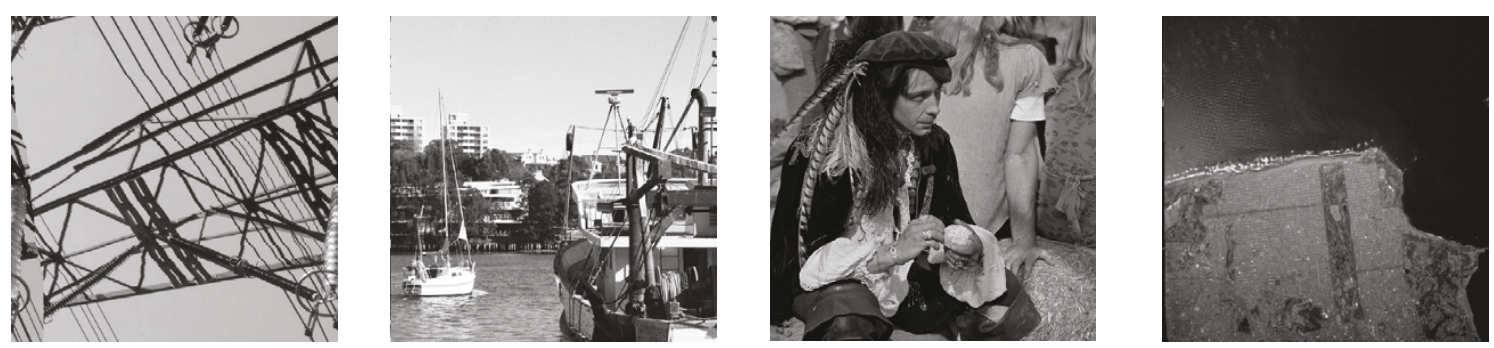

FIGURE 9: Image files in the training data set (Fold 3). 

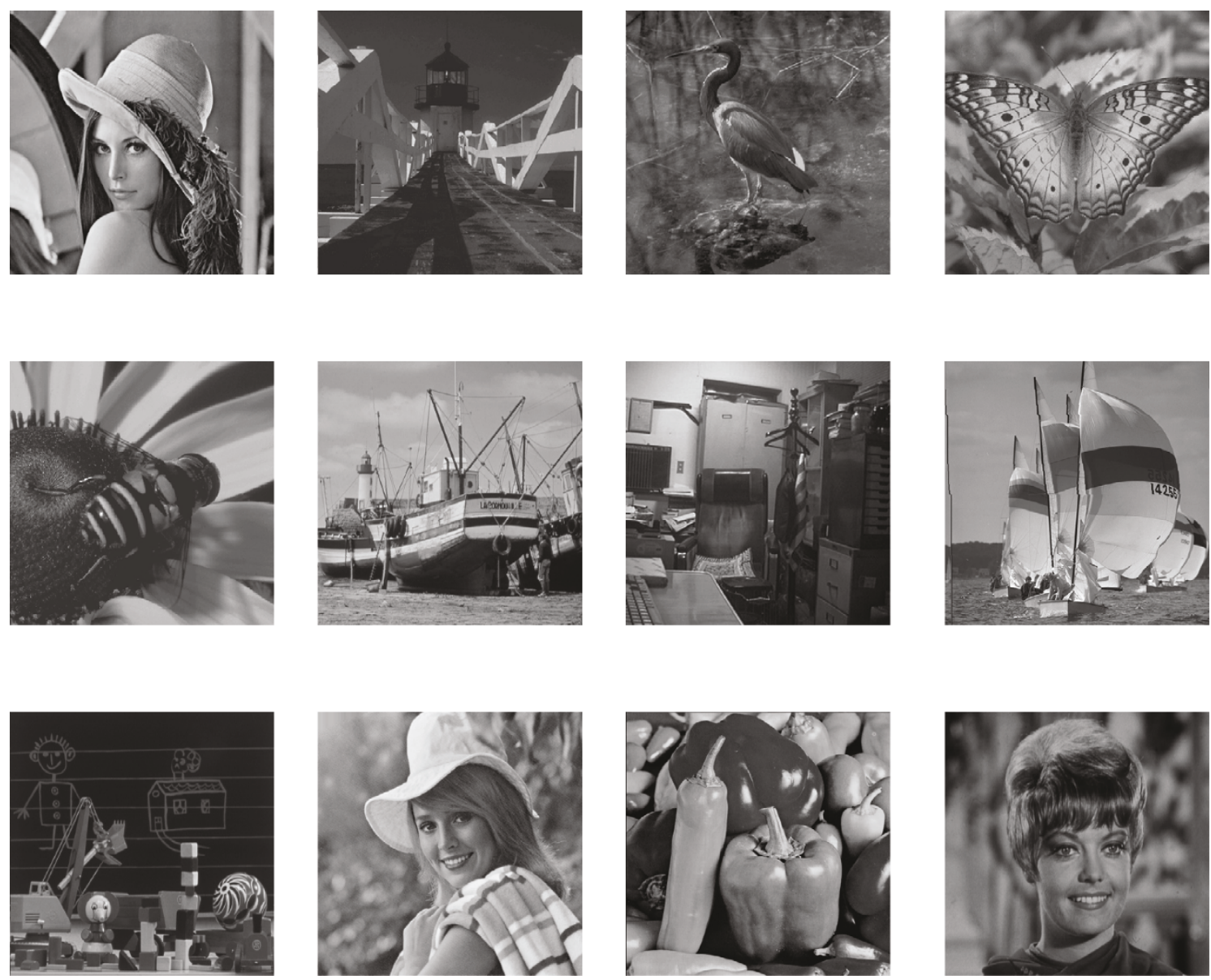

FIgUre 10: Image files in the test data set (TDS).

TABle 1: Training and test data file sets.

\begin{tabular}{lcr}
\hline File sets & Name of the files in the sets & $L_{\text {IBV }}$ \\
\hline Fold 1 & F1, F2, F3, F4, F5, F6, F7 & $8 \times 8=64$ \\
Fold 2 & F8, F11, F12, F13, F16, F38, F40 & $8 \times 8=64$ \\
Fold 3 & F22, F47, F64, F69 & $8 \times 8=64$ \\
TDA & All image files (TDS is excluded) & $16 \times 16=256$ \\
\hline Test data set (TDS) & Lenna, F14, F15, F24, F28, F39, F41, F48, F60, F61, F65, F73 & $8 \times 8=64$ \\
& & $16 \times 16=256$ \\
\hline
\end{tabular}

TABLE 2: Bit allocation table for the first group of experiments.

\begin{tabular}{|c|c|c|c|c|}
\hline File sets & CEPB size & Number of bits required & CEPB size (with clustering) & Number of bits required (with clustering) \\
\hline \multirow{3}{*}{ Fold 1} & $\mathrm{CEB}=31<2^{5}$ & $\mathrm{CEB}=5$ & $\mathrm{CEB}=32<2^{5}$ & $\mathrm{CEB}=5$ \\
\hline & $\mathrm{CPB}=15607<2^{14}$ & $\mathrm{CPB}=14$ & $\mathrm{CPB}=4096<2^{12}$ & $\mathrm{CPB}=12$ \\
\hline & & $\mathrm{BSC}=5$ & & $\mathrm{BSC}=5$ \\
\hline \multirow{3}{*}{ Fold 2} & $\mathrm{CEB}=32=2^{5}$ & $\mathrm{CEB}=5$ & $\mathrm{CEB}=32<2^{5}$ & $\mathrm{CEB}=5$ \\
\hline & $\mathrm{CPB}=15811<2^{14}$ & $\mathrm{CPB}=14$ & $\mathrm{CPB}=4096<2^{12}$ & $\mathrm{CPB}=12$ \\
\hline & & $\mathrm{BSC}=5$ & & $\mathrm{BSC}=5$ \\
\hline \multirow{3}{*}{ Fold 3} & $\mathrm{CEB}=32=2^{5}$ & $\mathrm{CEB}=5$ & $\mathrm{CEB}=32<2^{5}$ & $\mathrm{CEB}=5$ \\
\hline & $\mathrm{CPB}=13684<2^{14}$ & $\mathrm{CPB}=14$ & $\mathrm{CPB}=4096<2^{12}$ & $\mathrm{CPB}=12$ \\
\hline & & $\mathrm{BSC}=5$ & & $\mathrm{BSC}=5$ \\
\hline
\end{tabular}


TABLE 3: Evaluation results of Fold 1.

\begin{tabular}{lccccccc}
\hline Image file name & Block size & Bit per pixel (bpp) & Compression ratio (CR) & MSE & PSNR (dB) & MSE (filtered) & PSNR (dB) (filtered) \\
\hline Lenna & $8 \times 8$ & 0,375 & 21,33 & 0,00130 & 28,93 & 0,00110 & 29,66 \\
F14 & $8 \times 8$ & 0,375 & 21,33 & 0,00059 & 32,23 & 0,00052 & 32,80 \\
F15 & $8 \times 8$ & 0,375 & 21,33 & 0,00087 & 30,61 & 0,00078 & 31,07 \\
F24 & $8 \times 8$ & 0,375 & 21,33 & 0,00150 & 28,12 & 0,00140 & 28,59 \\
F28 & $8 \times 8$ & 0,375 & 21,33 & 0,00077 & 31,09 & 0,00071 & 31,43 \\
F39 & $8 \times 8$ & 0,375 & 21,33 & 0,00150 & 28,13 & 0,00140 & 28,53 \\
F41 & $8 \times 8$ & 0,375 & 21,33 & 0,00073 & 31,31 & 0,00058 & 32,31 \\
F48 & $8 \times 8$ & 0,375 & 21,33 & 0,00150 & 28,27 & 0,00160 & 28,08 \\
F60 & $8 \times 8$ & 0,375 & 21,33 & 0,00082 & 30,83 & 0,00072 & 31,42 \\
F61 & $8 \times 8$ & 0,375 & 21,33 & 0,00093 & 30,29 & 0,00084 & 30,74 \\
F65 & $8 \times 8$ & 0,375 & 21,33 & 0,00110 & 29,70 & 0,00093 & 30,28 \\
F73 & $8 \times 8$ & 0,375 & 21,33 & 0,00046 & 33,36 & 0,00038 & 34,11 \\
\hline Average & $8 \times 8$ & 0,375 & 21,33 & 0,00101 & 30,24 & 0,00091 & 30,75 \\
\hline
\end{tabular}

TABLE 4: Evaluation results of Fold 2.

\begin{tabular}{lccccccc}
\hline Image file name & Block size & Bit per pixel (bpp) & Compression ratio (CR) & MSE & PSNR (dB) & MSE (filtered) & PSNR (dB) (filtered) \\
\hline Lenna & $8 \times 8$ & 0,375 & 21,33 & 0,00140 & 28,45 & 0,00110 & 29,47 \\
F14 & $8 \times 8$ & 0,375 & 21,33 & 0,00073 & 31,36 & 0,00058 & 32,32 \\
F15 & $8 \times 8$ & 0,375 & 21,33 & 0,00097 & 30,11 & 0,00085 & 30,70 \\
F24 & $8 \times 8$ & 0,375 & 21,33 & 0,00180 & 27,47 & 0,00150 & 28,16 \\
F28 & $8 \times 8$ & 0,375 & 21,33 & 0,00087 & 30,59 & 0,00077 & 31,11 \\
F39 & $8 \times 8$ & 0,375 & 21,33 & 0,00180 & 27,50 & 0,00150 & 28,21 \\
F41 & $8 \times 8$ & 0,375 & 21,33 & 0,00089 & 30,48 & 0,00065 & 31,87 \\
F48 & $8 \times 8$ & 0,375 & 21,33 & 0,00180 & 27,44 & 0,00170 & 27,74 \\
F60 & $8 \times 8$ & 0,375 & 21,33 & 0,00110 & 29,73 & 0,00090 & 30,44 \\
F61 & $8 \times 8$ & 0,375 & 21,33 & 0,00099 & 30,03 & 0,00079 & 31,02 \\
F65 & $8 \times 8$ & 0,375 & 21,33 & 0,00120 & 29,15 & 0,00099 & 30,04 \\
F73 & $8 \times 8$ & 0,375 & 21,33 & 0,00053 & 32,73 & 0,00041 & 33,79 \\
\hline Average & $8 \times 8$ & 0,375 & 21,33 & 0,00117 & 29,59 & 0,00098 & 30,41 \\
\hline
\end{tabular}

TABLE 5: Evaluation results of Fold 3.

\begin{tabular}{lccccccc}
\hline Image file name & Block size & Bit per pixel (bpp) & Compression ratio (CR) & MSE & PSNR (dB) & MSE (filtered) & PSNR (dB) (filtered) \\
\hline Lenna & $8 \times 8$ & 0,375 & 21,33 & 0,00140 & 28,46 & 0,00110 & 29,45 \\
F14 & $8 \times 8$ & 0,375 & 21,33 & 0,00075 & 31,24 & 0,00060 & 32,21 \\
F15 & $8 \times 8$ & 0,375 & 21,33 & 0,00100 & 29,88 & 0,00087 & 30,59 \\
F24 & $8 \times 8$ & 0,375 & 21,33 & 0,00180 & 27,42 & 0,00150 & 28,13 \\
F28 & $8 \times 8$ & 0,375 & 21,33 & 0,00089 & 30,46 & 0,00078 & 31,03 \\
F39 & $8 \times 8$ & 0,375 & 21,33 & 0,00180 & 27,44 & 0,00150 & 28,14 \\
F41 & $8 \times 8$ & 0,375 & 21,33 & 0,00090 & 30,45 & 0,00067 & 31,73 \\
F48 & $8 \times 8$ & 0,375 & 21,33 & 0,00180 & 27,45 & 0,00170 & 27,67 \\
F60 & $8 \times 8$ & 0,375 & 21,33 & 0,00110 & 29,55 & 0,00093 \\
F61 & $8 \times 8$ & 0,375 & 21,33 & 0,00099 & 30,02 & 0,00080 & 30,30 \\
F65 & $8 \times 8$ & 0,375 & 21,33 & 0,00120 & 29,17 & 0,00098 \\
F73 & $8 \times 8$ & 0,375 & 21,33 & 0,00057 & 32,40 & 0,00044 & 30,93 \\
\hline Average & $8 \times 8$ & 0,375 & 21,33 & 0,00118 & 29,50 & 0,00099 & 30,07 \\
\hline
\end{tabular}


TABLE 6: Evaluation results of Fold 1 (with clustering).

\begin{tabular}{lccccccc}
\hline Image file name & Block size & Bit per pixel (bpp) & Compression ratio (CR) & MSE & PSNR (dB) & MSE (filtered) & PSNR (dB) (filtered) \\
\hline Lenna & $8 \times 8$ & 0,3438 & 23,27 & 0,00140 & 28,46 & 0,00120 & 29,16 \\
F14 & $8 \times 8$ & 0,3438 & 23,27 & 0,00067 & 31,71 & 0,00060 & 32,22 \\
F15 & $8 \times 8$ & 0,3438 & 23,27 & 0,00095 & 30,19 & 0,00086 & 30,64 \\
F24 & $8 \times 8$ & 0,3438 & 23,27 & 0,00170 & 27,68 & 0,00150 & 28,17 \\
F28 & $8 \times 8$ & 0,3438 & 23,27 & 0,00084 & 30,71 & 0,00077 & 31,10 \\
F39 & $8 \times 8$ & 0,3438 & 23,27 & 0,00170 & 27,63 & 0,00160 & 28,06 \\
F41 & $8 \times 8$ & 0,3438 & 23,27 & 0,00082 & 30,86 & 0,00067 & 31,72 \\
F48 & $8 \times 8$ & 0,3438 & 23,27 & 0,00170 & 27,71 & 0,00170 & 27,68 \\
F60 & $8 \times 8$ & 0,3438 & 23,27 & 0,00100 & 29,95 & 0,00090 & 30,44 \\
F61 & $8 \times 8$ & 0,3438 & 23,27 & 0,00092 & 30,32 & 0,00082 & 30,86 \\
F65 & $8 \times 8$ & 0,3438 & 23,27 & 0,00120 & 29,19 & 0,00110 & 29,74 \\
F73 & $8 \times 8$ & 0,3438 & 23,27 & 0,00050 & 32,97 & 0,00043 & 33,67 \\
\hline Average & $8 \times 8$ & 0,3438 & 23,27 & 0,00112 & 29,78 & 0,00101 & 30,29 \\
\hline
\end{tabular}

TABLE 7: Evaluation results of Fold 2 (with clustering).

\begin{tabular}{lccccccc}
\hline Image file name & Block size & Bit per pixel (bpp) & Compression ratio (CR) & MSE & PSNR (dB) & MSE (filtered) & PSNR (dB) (filtered) \\
\hline Lenna & $8 \times 8$ & 0,3438 & 23,27 & 0,00160 & 28,06 & 0,00130 & 28,86 \\
F14 & $8 \times 8$ & 0,3438 & 23,27 & 0,00081 & 30,91 & 0,00067 & 31,72 \\
F15 & $8 \times 8$ & 0,3438 & 23,27 & 0,00110 & 29,67 & 0,00095 & 30,20 \\
F24 & $8 \times 8$ & 0,3438 & 23,27 & 0,00200 & 27,07 & 0,00170 & 27,68 \\
F28 & $8 \times 8$ & 0,3438 & 23,27 & 0,00095 & 30,21 & 0,00084 & 30,73 \\
F39 & $8 \times 8$ & 0,3438 & 23,27 & 0,00200 & 26,97 & 0,00170 & 27,60 \\
F41 & $8 \times 8$ & 0,3438 & 23,27 & 0,00097 & 30,13 & 0,00076 & 31,17 \\
F48 & $8 \times 8$ & 0,3438 & 23,27 & 0,00200 & 26,89 & 0,00190 & 27,19 \\
F60 & $8 \times 8$ & 0,3438 & 23,27 & 0,00110 & 29,42 & 0,00099 & 30,04 \\
F61 & $8 \times 8$ & 0,3438 & 23,27 & 0,00110 & 29,66 & 0,00089 & 30,46 \\
F65 & $8 \times 8$ & 0,3438 & 23,27 & 0,00140 & 28,64 & 0,00120 & 29,36 \\
F73 & $8 \times 8$ & 0,3438 & 23,27 & 0,00057 & 32,37 & 0,00047 & 33,24 \\
\hline Average & $8 \times 8$ & 0,3438 & 23,27 & 0,00130 & 29,17 & 0,00111 & 29,85 \\
\hline
\end{tabular}

TABLE 8: Evaluation results of Fold 3 (with clustering).

Image file name Block size $\quad$ Bit per pixel (bpp) Compression ratio (CR) $\quad$ MSE $\quad$ PSNR (dB) MSE (filtered) PSNR (dB) (filtered)

\begin{tabular}{llllllll}
\hline Lenna & $8 \times 8$ & 0,3438 & 23,27 & 0,00150 & 28,30 & 0,00120 & 29,16 \\
F14 & $8 \times 8$ & 0,3438 & 23,27 & 0,00081 & 30,90 & 0,00066 & 31,75 \\
F15 & $8 \times 8$ & 0,3438 & 23,27 & 0,00110 & 29,67 & 0,00094 & 30,24 \\
F24 & $8 \times 8$ & 0,3438 & 23,27 & 0,00190 & 27,15 & 0,00170 & 27,78 \\
F28 & $8 \times 8$ & 0,3438 & 23,27 & 0,00094 & 30,25 & 0,00053 & 30,76 \\
F39 & $8 \times 8$ & 0,3438 & 23,27 & 0,00190 & 27,12 & 0,00170 & 27,75 \\
F41 & $8 \times 8$ & 0,3438 & 23,27 & 0,00096 & 30,17 & 0,00074 & 31,26 \\
F48 & $8 \times 8$ & 0,3438 & 23,27 & 0,00200 & 27,08 & 0,00180 & 27,35 \\
F60 & $8 \times 8$ & 0,3438 & 23,27 & 0,00110 & 29,48 & 0,00096 & 30,15 \\
F61 & $8 \times 8$ & 0,3438 & 23,27 & 0,00110 & 29,76 & 0,00087 & 30,57 \\
F65 & $8 \times 8$ & 0,3438 & 23,27 & 0,00130 & 28,90 & 0,00110 & 29,65 \\
F73 & $8 \times 8$ & 0,3438 & 23,27 & 0,00058 & 32,30 & 0,00047 & 33,20 \\
\hline Average & $8 \times 8$ & 0,3438 & 23,27 & 0,00127 & 29,26 & 0,00106 & 29,97 \\
\hline
\end{tabular}


TABLE 9: Bit allocation table for the second group of experiments.

\begin{tabular}{|c|c|c|c|c|}
\hline File sets & CEPB size & Number of bits required & CEPB size (clustered) & Number of bits required \\
\hline \multirow{3}{*}{ TDA } & $\mathrm{CEB}=171<2^{8}$ & $\mathrm{CEB}=8$ & $\mathrm{CEB}=32<2^{5}$ & $\mathrm{CEB}=5$ \\
\hline & $\mathrm{CPB}=54306<2^{16}$ & $\mathrm{CPB}=16$ & $\mathrm{CPB}=13312<2^{14}$ & $\mathrm{CPB}=14$ \\
\hline & & $\mathrm{BSC}=5$ & & $\mathrm{BSC}=5$ \\
\hline
\end{tabular}

TABLE 10: Evaluation results of the second group of experiments.

\begin{tabular}{|c|c|c|c|c|c|c|c|}
\hline Image file name & Block size & Bit per pixel (bpp) & Compression ratio (CR) & MSE & PSNR (dB) & MSE (filtered) & PSNR (dB) (filtered) \\
\hline Lenna & $16 \times 16$ & 0,2266 & 70,62 & 0,00300 & 25,20 & 0,00260 & 25,87 \\
\hline F14 & $16 \times 16$ & 0,2266 & 70,62 & 0,00150 & 28,35 & 0,00130 & 28,87 \\
\hline F15 & $16 \times 16$ & 0,2266 & 70,62 & 0,00180 & 27,43 & 0,00160 & 27,88 \\
\hline F24 & $16 \times 16$ & 0,2266 & 70,62 & 0,00350 & 24,57 & 0,00310 & 25,06 \\
\hline F28 & $16 \times 16$ & 0,2266 & 70,62 & 0,00150 & 28,20 & 0,00130 & 28,77 \\
\hline F39 & $16 \times 16$ & 0,2266 & 70,62 & 0,00340 & 24,73 & 0,00300 & 25,17 \\
\hline F41 & $16 \times 16$ & 0,2266 & 70,62 & 0,00190 & 27,18 & 0,00160 & 27,83 \\
\hline F48 & $16 \times 16$ & 0,2266 & 70,62 & 0,00310 & 25,02 & 0,00310 & 25,05 \\
\hline F60 & $16 \times 16$ & 0,2266 & 70,62 & 0,00180 & 27,38 & 0,00150 & 28,15 \\
\hline F61 & $16 \times 16$ & 0,2266 & 70,62 & 0,00220 & 26,61 & 0,00190 & 27,17 \\
\hline F65 & $16 \times 16$ & 0,2266 & 70,62 & 0,00250 & 25,94 & 0,00220 & 26,62 \\
\hline F73 & $16 \times 16$ & 0,2266 & 70,62 & 0,00110 & 29,58 & 0,00092 & 30,36 \\
\hline Average & $16 \times 16$ & 0,2266 & 70,62 & 0,00228 & 26,68 & 0,00201 & 27,23 \\
\hline
\end{tabular}

TABLE 11: Evaluation results of the second group of experiments (with clustering).

\begin{tabular}{|c|c|c|c|c|c|c|c|}
\hline Image file name & Block size & Bit per pixel (bpp) & Compression ratio (CR) & MSE & PSNR (dB) & MSE (filtered) & PSNR (dB) (filtered) \\
\hline Lenna & $16 \times 16$ & 0,1875 & 85,33 & 0,00320 & 24,95 & 0,00280 & 25,57 \\
\hline F14 & $16 \times 16$ & 0,1875 & 85,33 & 0,00160 & 28,00 & 0,00140 & 28,46 \\
\hline F15 & $16 \times 16$ & 0,1875 & 85,33 & 0,00190 & 27,16 & 0,00180 & 27,56 \\
\hline F24 & $16 \times 16$ & 0,1875 & 85,33 & 0,00380 & 24,25 & 0,00340 & 24,73 \\
\hline F28 & $16 \times 16$ & 0,1875 & 85,33 & 0,00160 & 27,99 & 0,00140 & 28,54 \\
\hline F39 & $16 \times 16$ & 0,1875 & 85,33 & 0,00360 & 24,39 & 0,00330 & 24,81 \\
\hline F41 & $16 \times 16$ & 0,1875 & 85,33 & 0,00200 & 26,92 & 0,00180 & 27,45 \\
\hline F48 & $16 \times 16$ & 0,1875 & 85,33 & 0,00350 & 24,53 & 0,00340 & 24,69 \\
\hline F60 & $16 \times 16$ & 0,1875 & 85,33 & 0,00200 & 27,03 & 0,00170 & 27,68 \\
\hline F61 & $16 \times 16$ & 0,1875 & 85,33 & 0,00230 & 26,31 & 0,00210 & 26,80 \\
\hline F65 & $16 \times 16$ & 0,1875 & 85,33 & 0,00280 & 25,52 & 0,00240 & 26,16 \\
\hline F73 & $16 \times 16$ & 0,1875 & 85,33 & 0,00120 & 29,29 & 0,00100 & 29,98 \\
\hline Average & $16 \times 16$ & 0,1875 & 85,33 & 0,00246 & 26,36 & 0,00221 & 26,87 \\
\hline
\end{tabular}


TABLE 12: Evaluation results (overall).

\begin{tabular}{|c|c|c|c|c|c|c|c|c|}
\hline Group of experiment & Fold & Block size & $\begin{array}{c}\text { Bit per pixel } \\
\text { (bpp) }\end{array}$ & $\begin{array}{c}\text { Compression } \\
\text { ratio }(\mathrm{CR}) \\
\end{array}$ & MSE & PSNR (dB) & $\begin{array}{c}\text { MSE } \\
\text { (filtered) }\end{array}$ & $\begin{array}{c}\text { PSNR (dB) } \\
\text { (filtered) }\end{array}$ \\
\hline \multirow{3}{*}{1} & Fold 1 & \multirow{3}{*}{$8 \times 8$} & \multirow{3}{*}{0,3750} & \multirow{3}{*}{21,33} & 0,00101 & 30,24 & 0,00091 & 30,75 \\
\hline & Fold 2 & & & & 0,00117 & 29,59 & 0,00098 & 30,41 \\
\hline & Fold 3 & & & & 0,00118 & 29,50 & 0,00099 & 30,32 \\
\hline Average & & $8 \times 8$ & 0,3750 & 21,33 & 0,00112 & 29,78 & 0,00096 & 30,49 \\
\hline \multirow{3}{*}{1} & Fold 1 & \multirow{3}{*}{$8 \times 8$} & \multirow{3}{*}{0,3438} & \multirow{3}{*}{23,27} & 0,00112 & 29,78 & 0,00101 & 30,29 \\
\hline & Fold 2 & & & & 0,00130 & 29,17 & 0,00111 & 29,85 \\
\hline & Fold 3 & & & & 0,00127 & 29,26 & 0,00106 & 29,97 \\
\hline Average & & $8 \times 8$ & 0,3438 & 23,27 & 0,00123 & 29,40 & 0,00106 & 30,04 \\
\hline \multirow{2}{*}{2} & & \multirow{2}{*}{$16 \times 16$} & 0,2266 & 70,62 & 0,00228 & 26,68 & 0,00201 & 27,23 \\
\hline & & & 0,1875 & 85,33 & 0,00246 & 26,36 & 0,00221 & 26,87 \\
\hline
\end{tabular}

to represent the $8 \times 8$ blocks of the images. In this case, the compression ratio will be computed as follows:

$$
\begin{gathered}
\mathrm{CR}=\frac{\text { bit }_{\text {original }}}{\text { bit }_{\text {reconstructed }}}=\frac{(8 \times 8) \times 8 \text { bits }}{(5+14+5) \text { bits }}=\frac{512}{24}=21,3333, \\
\text { or bpp }=\frac{\sqrt{L_{\mathrm{IBV}}}}{\mathrm{CR}}=\frac{\sqrt{8 \times 8}}{21,33}=0,3750 .
\end{gathered}
$$

The number of classified energy and pattern blocks and the required number of bits to represent each $8 \times 8$ block of the images are shown in Table 2.

The evaluation results of Fold 1, Fold 2, and Fold 3 are presented in Tables 3, 4, and 5, respectively.

The same experiments are repeated with the new resized CEPB obtained after the clustering algorithm. At the end of the clustering process the sizes of the $\mathrm{CEB}$ and $\mathrm{CPB}$ are reduced and the number of classified energy and pattern blocks is determined as $2^{5}$ and $2^{12}$, respectively. Thus, an 8 $\times 8$ image blocks can be represented by 22 bits in total as given in Table 2. The evaluation results of Fold 1, Fold 2, and Fold 3 with clustering are presented in Tables 6, 7, and 8, respectively.

In the second group of experiments the total number of bits required to represent the $16 \times 16$ blocks for each original image file is $(16 \times 16) \times 8$ bits $=2048$ bits. In these experiments the size of the CEPB is determined and fixed for all folds (3 Folds) by adjusting the PCC. Thus, total numbers of classified energy and pattern blocks are determined in the range of $2^{8}$ and $2^{16}$ in the CEB and CPB sets, respectively. $N_{\mathrm{IE}}, N_{\mathrm{IP}}$ and BSC are represented by 8 bits, 16 bits, and 5 bits, respectively. To represent the $16 \times 16$ blocks of the images, 29 bits are required in total. In this case, the compression ratio will be computed as follows,

$$
\begin{aligned}
& \mathrm{CR}=\frac{\text { bit }_{\text {original }}}{\text { bit }_{\text {reconstructed }}}=\frac{(16 \times 16) \times 8 \text { bits }}{(8+16+5) \text { bits }}=\frac{2048}{29}=70,6207, \\
& \text { or bpp }=\frac{\sqrt{\mathrm{L}_{\mathrm{IBV}}}}{\mathrm{CR}}=\frac{\sqrt{16 \times 16}}{70,6207}=0,2266 .
\end{aligned}
$$

The number of classified energy and pattern blocks and the number of bits required to represent each $16 \times 16$ blocks of the images are given in Table 9. The evaluation results of the second group of experiments are presented in Table 10.

In order to reach lower bit rates we also established the same experiments using an efficient clustering algorithm. At the end of the clustering process the sizes of the $\mathrm{CEB}$ and $\mathrm{CPB}$ are reduced and the number of classified energy and pattern blocks is determined as $2^{5}$ and $2^{14}$, respectively. In this case, $16 \times 16$ image blocks can be represented by 24 bits in total as given in Table 9. The evaluation results of the second group of experiments with clustering are presented in Table 11.

Overall evaluation performance of the method including the average results of the first and second groups of experiments is summarized in Table 12 .

In order to show the performance of the proposed method, some of the original and reconstructed versions of the image are exhibited in Figures 11 and 12 for the first group of experiments and the second group of experiments, respectively. It is clearly understood from the figures and the evaluation results given in the tables that, the performance of the proposed method depends on the size of the CEPB associated with the size of the $L_{\mathrm{IBV}}$. If the size of the CEPB is highly reduced or the size of the $L_{\mathrm{IBV}}$ is increased in order to achieve higher compression ratios or lower bit rates, the performance of the method is getting worse and the blocking effect is also getting visible. Even in this case, it is remarkable that the PSNR levels are not affected dramatically and they do not drop below $27 \mathrm{~dB}$ at higher compression ratios $(0,1877 \mathrm{bpp}$ or $85,33: 1$ compression ratio on the average). It is also noted that there is almost no difference in terms of PSNR level between the images compressed at $70,62: 1(27,23 \mathrm{~dB})$ and $85,33: 1(26,87 \mathrm{~dB})$. The blocking effect in the reconstructed images at various compression ratios and the results of the filtering process are illustrated in Figures 13(A) and 13(B).

\section{Conclusion and Future Works}

In this paper, a new image compression algorithm based on the classified energy and pattern block (CEPB) sets is 

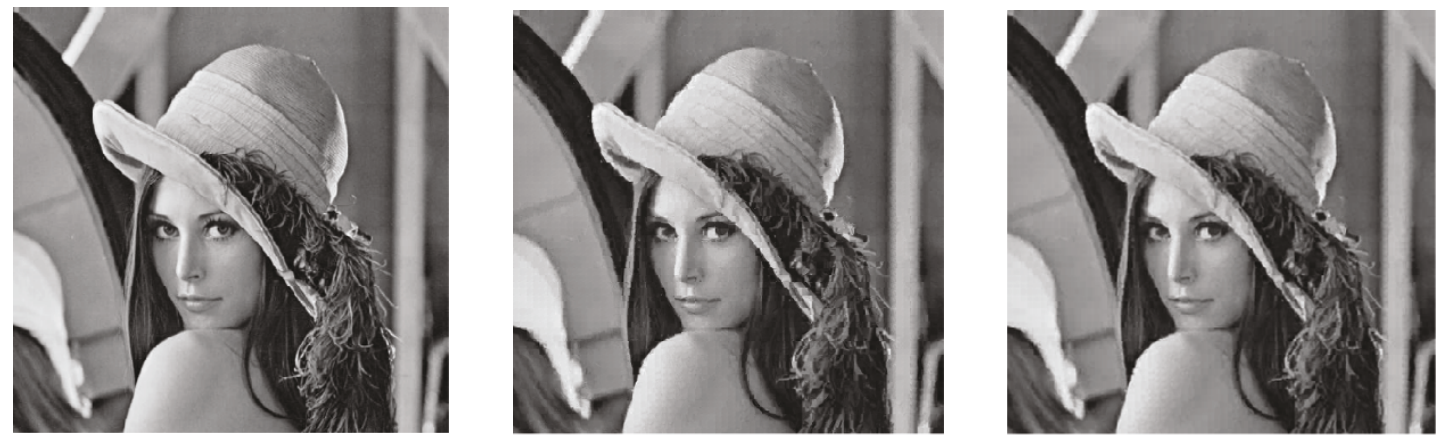

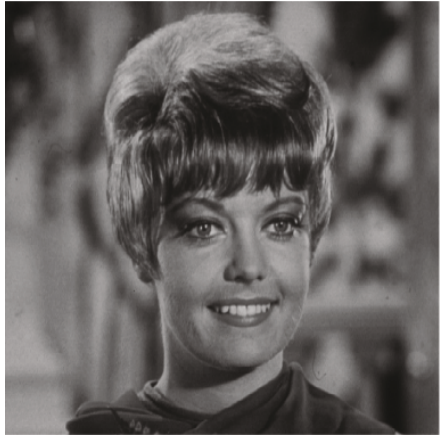

(a)

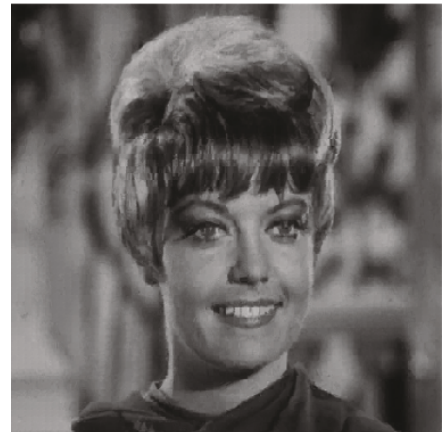

(b)

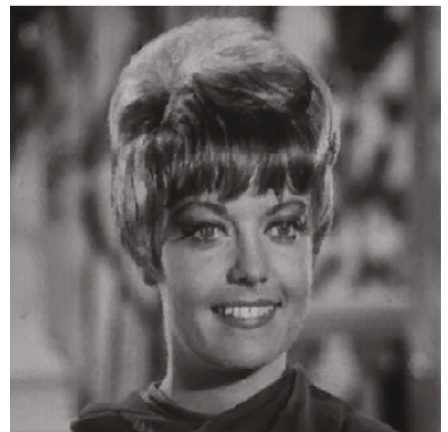

(c)

FIGURE 11: (a) Original, (b) reconstructed, and (c) filtered versions of the images (Lenna and F73) for the 1st group of experiments (Fold 1). Lenna: PSNR = 28,93 and 29,66 (filtered), F73: PSNR = 33,36 and 34,11 (filtered) $(\mathrm{CR}=21,33)$.
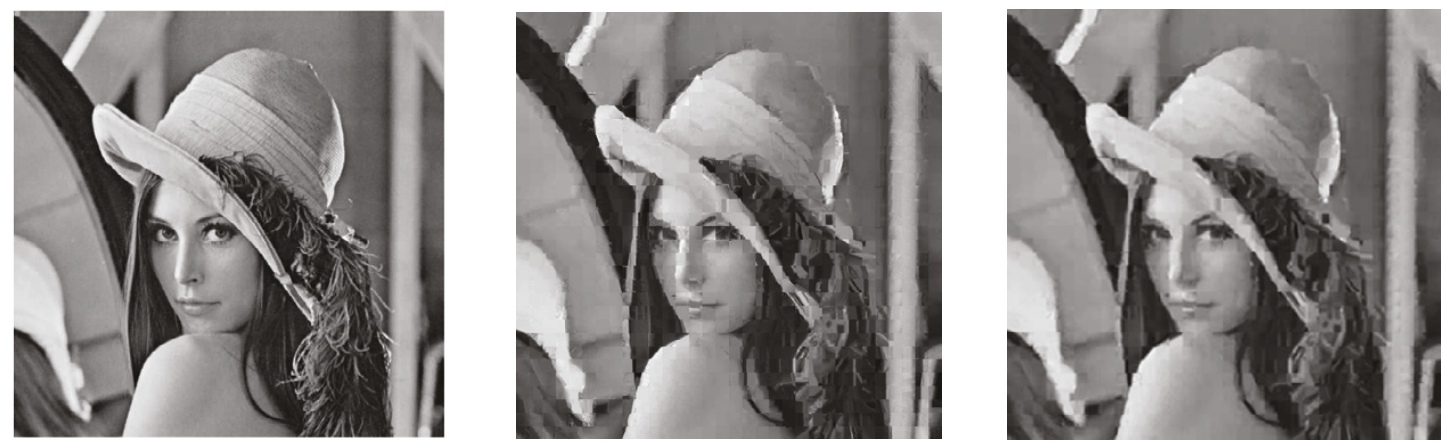

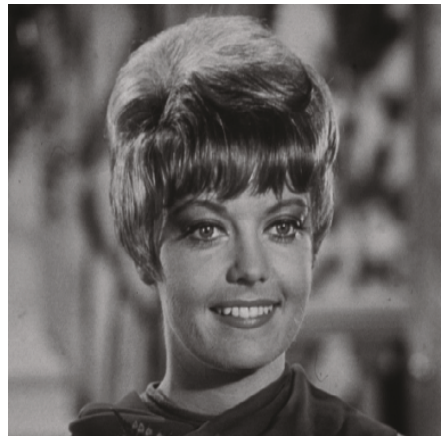

(a)

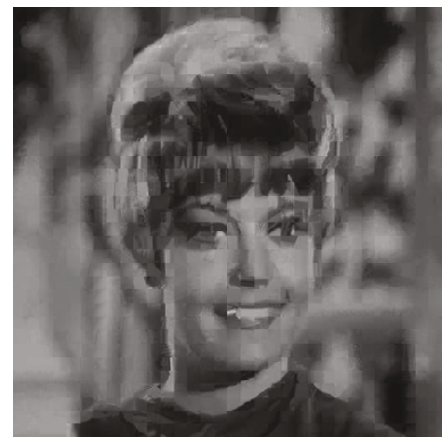

(b)

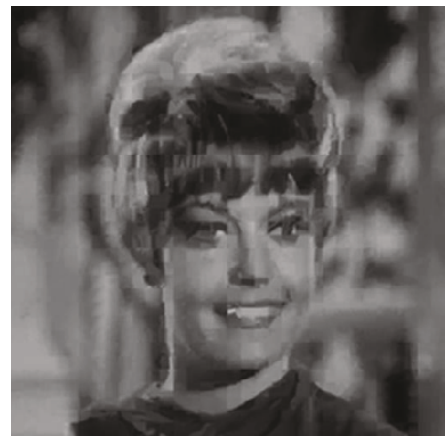

(c)

Figure 12: (a) Original, (b) reconstructed, and (c) filtered versions of the images (Lenna and F73) for the 2nd group of experiments. Lenna: PSNR $=25,20$ and 25,87 (filtered), F73: PSNR $=29,58$ and 30,36 (filtered) $(\mathrm{CR}=70,62)$. 

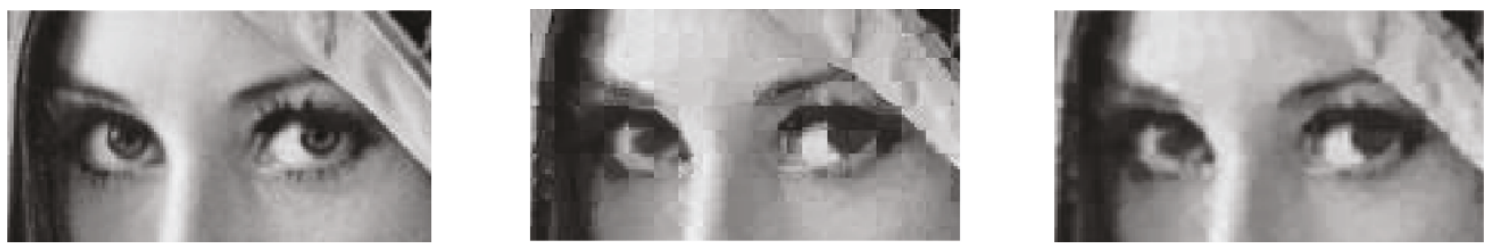

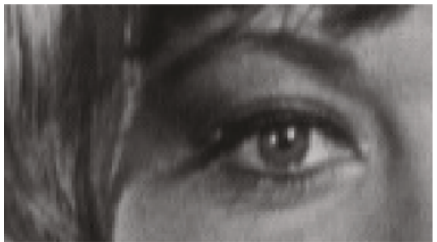

(a)
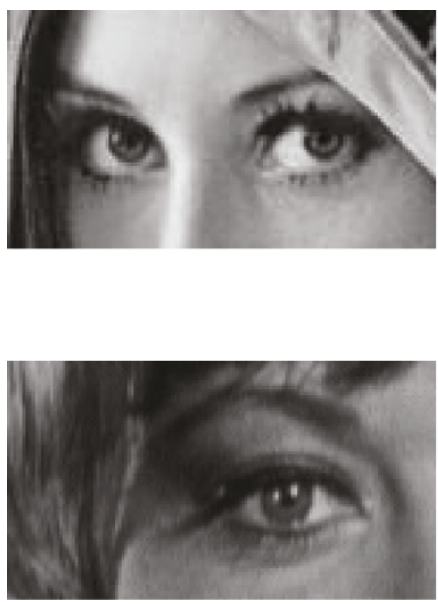

(a)

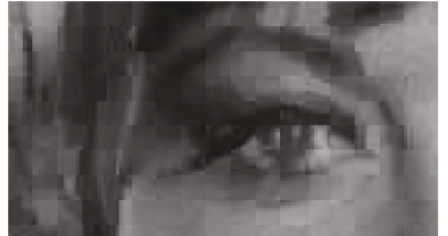

(b)

(A)
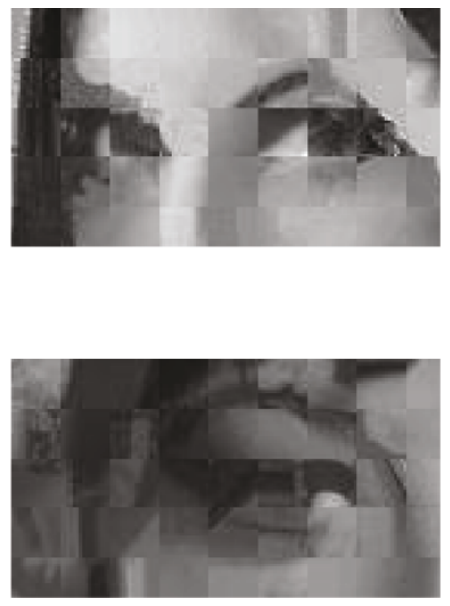

(b)

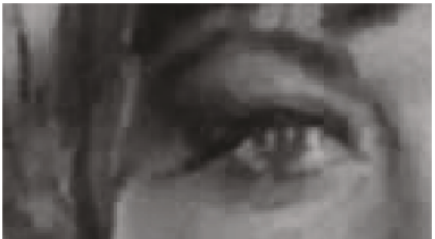

(c)
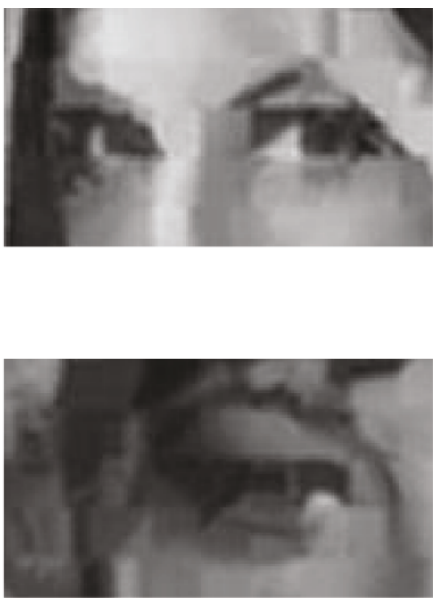

(c)

(B)

FIgURE 13: (A) Cropped (a) original, (b) reconstructed, and (c) filtered versions of the images (Lenna and F73) for the 1st group of experiments (Fold 1). Lenna: PSNR = 28,93 and 29,66 (filtered), F73: PSNR = 33,36 and 34,11 (filtered) $($ CR $=21,33$ ). (B) Cropped (a) original, (b) reconstructed, and (c) filtered versions of the images (Lenna and F73) for the 2nd group of experiments. Lenna: PSNR $=25,20$ and 25,87 (filtered), F73: PSNR = 29,58 and 30,36 (filtered) $(\mathrm{CR}=70,62)$.

proposed. In the method, first the CEB and CPB sets are constructed and any image data can be reconstructed block by block using a block scaling coefficient and the index numbers of the classified energy and pattern blocks placed in the CEB and CPB. The CEB and CPB sets are constructed for different sizes of image blocks such as 8 by 8 or 16 by 16 with respect to different compression ratios desired.

At the end of a series of the experimental works, the evaluation results show that the proposed method provides high compression ratios such as $21,33: 1,85,33: 1$ while preserving the image quality at $27-30.5 \mathrm{~dB}$ level on the average. When the compression ratio versus image quality (PSNR) results in the proposed method compared to the other works [47], it seems that the method is superior to the DCT and DWT particularly at low bit rates or high compression ratios.
For the time being, the performance of the newly proposed method is measured using PSNR and MSE metrics and in the next paper on the comparative results of this work, the other quality assessment metrics such as SSIM will also be considered.

In our future works we will be focused on better designed CEB and CPB in order to increase the level of the PSNR while reducing the number of bits required representing the image blocks. It was also concluded that the edge artifacts at the boundaries of the reconstructed blocks affects the quality of the reconstructed images. We currently are working on removing or smoothing these artifacts using some postprocessing and filtering algorithms. As a starting point the 2D Savitzky-Golay smoothing filter is applied on the reconstructed images and the PSNR levels are improved 0.5-1 dB compared to plain version of the method. 
In terms of computational complexity, none of the implementations we employ are optimized for execution speed. Even the size of the CEPB is reduced and the speed of the algorithms is increased with an efficient clustering algorithm this attempt results in degraded image caused by blocking effect in the reconstruction stage especially at low bit rates. Our next work will also consider speeding up the main procedure, in particular the encoding stage, with less degradation.

It should be noted that we are also working on the algorithms which select optimum block size length (variable) instead of fixed in order to get higher compression ratio in overall with less degradation caused by blocking effect. In these algorithms, the block size is adaptively changed so that it is increased in the plain or spatially redundant areas while it is decreased in the other regions which contain the detailed information. In order to obtain more precise results, additional tests with different image sets containing biomedical (ultrasound, cell), face, and fingerprint images will be performed. Furthermore, application-specific CEPBs will be constructed and the possible effects of these CEPBs on the test images from the same and the different application domains will be analyzed.

The improved version of the method will also contain the Huffman encoding part which provides better performance in terms of compression ratio. In our future works we are not only planning to present the results of improved version of the method but also planning to compare the results to the other methods such as KLT, DCT, and wavelet-based methods considering the other quality assessment metrics which is more appropriate for HVS such as SSIM.

\section{Acknowledgments}

The work described in this paper was funded by the Isik University Scientific Research Fund (Project contract no. 10B301). The author would like to thank to Professor B. S. Yarman (Istanbul University, College of Engineering, Department of Electrical-Electronics Engineering), Assistant Professor Hakan Gurkan (Isik University, Engineering Faculty, Department of Electrical-Electronics Engineering), the researchers in the International Computer Science Institute (ICSI), Speech Group, University of California at Berkeley, CA, USA and the researchers in the SRI International, Speech Technology and Research (STAR) Laboratory, Menlo Park, CA, USA for many helpful discussions on this work during his postdoctoral fellow years. The author also would like to thank the anonymous reviewers for their valuable comments and suggestions which substantially improved the quality of this paper.

\section{References}

[1] R. C. Gonzales and R. E. Woods, Digital Image Processing, Pearson Education, Essex, UK, 2nd edition, 2004.

[2] D. Salomon, Data Compression: The Complete Reference, Springer, New York, NY, USA, 3rd edition, 2004.

[3] N. Jayant and P. Noll, Digital Coding of Waveforms: Principles and Applications to Speech and Video, Prentice-Hall, Englewood Cliffs, NJ, USA, 1984.
[4] N. Jayant, J. Johnston, and R. Safranek, "Signal compression based on models of human perception," Proceedings of the IEEE, vol. 81, no. 10, pp. 1385-1422, 1993.

[5] B. Z. Cihlar, S. Grgic, and D. Modric, "Coding techniques in multimedia communications," in Proceedings of the 2nd International Workshop on Image and Signal Processing (IWISP '95), pp. 24-32, 1995.

[6] T. Jolliffe, Principal Component Analysis, Springer Series in Statistics, Springer, New York, NY, USA, 1993.

[7] K. J. Anil, Fundamentals of Digital Image Processing, Prentice Hall, New York, NY, USA, 1989.

[8] M. Kirby and L. Sirovich, "Application of the Karhunen-Loeve procedure for the characterization of human faces," IEEE Transactions on Pattern Analysis and Machine Intelligence, vol. 12, no. 1, pp. 103-108, 1990.

[9] "Digital Compression and Coding of Continuous Tone Still Images," ISO/IEC IS 10918, 1991.

[10] G. K. Wallace, "The JPEG still picture compression standard," Communications of the ACM, vol. 34, no. 4, pp. 30-44, 1991.

[11] W. B. Pennebaker and J. L. Mitchell, JPEG Still Image Data Compression Standard, Van Nostrand Reinhold, New York, NY, USA, 1992.

[12] K. R. Rao and P. Yip, Discrete Cosine Transform: Algorithms, Advantages, and Applications, Academic Press, San Diego, Calif, USA, 1990.

[13] S. Bauer, B. Zovko-Cihlar, and M. Grgic, "The influence of impairments from digital compression of video signal on perceived picture quality," in Proceedings of the 3rd International Workshop on Image and Signal Processing (IWISP '96), pp. 245248, 1996.

[14] Z. Xiong, K. Ramchandran, M. T. Orchard, and YA. Q. Zhang, "A comparative study of DCT- and wavelet-based image coding," IEEE Transactions on Circuits and Systems for Video Technology, vol. 9, no. 5, pp. 692-695, 1999.

[15] I. Daubechies, Ten Lectures on Wavelets, SIAM, Philadelphia, Pa, USA, 1992.

[16] M. Vetterli and J. Kovacevic, Wavelets and Sub-Band Coding, Prentice-Hall, Englewood Cliffs, NJ, USA, 1995.

[17] M. Antonini, M. Barlaud, P. Mathieu, and I. Daubechies, "Image coding using wavelet transform," IEEE Transactions of Image Processing, vol. 1, no. 2, pp. 205-220, 1992.

[18] A. S. Lewis and G. Knowles, "Image compression using the 2D wavelet transform," IEEE Transactions of Image Processing, vol. 1, no. 2, pp. 244-250, 1992.

[19] S. G. Mallat, "Theory for multiresolution signal decomposition: the wavelet representation," IEEE Transactions on Pattern Analysis and Machine Intelligence, vol. 11, no. 7, pp. 674-693, 1989.

[20] J. M. Shapiro, "Embedded image coding using zerotrees of wavelet coefficients," IEEE Transactions on Signal Processing, vol. 41, no. 12, pp. 3445-3462, 1993.

[21] A. Said and W. A. Pearlman, "A new, fast, and efficient image codec based on set partitioning in hierarchical trees," IEEE Transactions on Circuits and Systems for Video Technology, vol. 6, no. 3, pp. 243-250, 1996.

[22] Z. Xiong, K. Ramchandran, and M. T. Orchard, "Spacefrequency quantization for wavelet image coding," IEEE Transactions on Image Processing, vol. 6, no. 5, pp. 677-693, 1997.

[23] E. S. Hong and R. E. Ladner, "Group testing for image compression," IEEE Transactions on Image Processing, vol. 11, no. 8, pp. 901-911, 2002.

[24] C. Christopoulos, "The jpeg2000 still image coding system: an overview," IEEE Transactions on Consumer Electronics, vol. 46, no. 4, pp. 1103-1127, 2000. 
[25] K. Pearson, "On lines and planes of closest fit to systems of points in space," Philosophical Magazine, vol. 6, no. 2, pp. 559-572, 1901.

[26] E. Oja, "Neural networks, principal components, and subspaces," International Journal on Neural Systems, vol. 1, pp. 61-68, 1989.

[27] W. W. Haher, Applied Numerical Linear Algebra, Prentice Hall, New York, NY, USA, 1988.

[28] T. D. Sanger, "Optimal unsupervised learning in a single-layer linear feedforward neural network," Neural Networks, vol. 2, no. 6, pp. 459-473, 1989.

[29] S. Y. Kung, K. I. Diamantaras, and J. S. Taur, "Adaptive principal component extraction (APEX) and applications," IEEE Transactions on Signal Processing, vol. 42, no. 5, pp. 1202-1271, 1994.

[30] H. M. Abbas and M. M. Fahmy, "Neural model for Karhumen-Loeve transform with application to adaptive image compression," IEE Proceedings, Part I: Communications, Speech and Vision, vol. 140, no. 2, pp. 135-143, 1993.

[31] S. Bannour and M. R. Azimi-Sadjadi, "Principal component extraction using recursive least squares learning," IEEE Transactions on Neural Networks, vol. 6, no. 2, pp. 457-469, 1995.

[32] A. C. S. Leung, K. W. Wong, and AH. C. Tsoi, "Recursive algorithms for principal component extraction," Network: Computation in Neural Systems, vol. 8, no. 3, pp. 323-334, 1997.

[33] A. Cichocki, W. Kasprzak, and W. Skarbek, "Adaptive learning algorithm for principal component analysis with partial data," in Proceedings of the 13th European Meeting on Cybernetics and Systems Research, pp. 1014-1019, 1996.

[34] ZE. Wang, Y. Lee, S. Fiori, C. S. Leung, and YI. S. Zhu, "An improved sequential method for principal component analysis," Pattern Recognition Letters, vol. 24, no. 9-10, pp. 1409-1415, 2003.

[35] S. Costa and S. Fiori, "Image compression using principal component neural networks," Image and Vision Computing, vol. 19, no. 9-10, pp. 649-668, 2001.

[36] C. Lv, Z. Liu, and Q. Zhao, "A flexible non-linear PCA encoder for still image compression," in Proceedings of the 7th IEEE International Conference on Computer and Information Technology (CIT '07), pp. 645-650, October 2007.

[37] K. M. Curtis, G. Neil, and V. Fotopoulos, "A hybrid fractal/ DCT image compression method," in Proceedings of the International Conference on Digital Signal Processing (DSP '02), pp. 1337-1340, 2002.

[38] N. N. Ponomarenko, K. O. Egiazarian, V. V. Lukin, and J. T. Astola, "High-quality DCT-based image compression using partition schemes," IEEE Signal Processing Letters, vol. 14, no. 2, pp. 105-108, 2007.

[39] S. D. Kulkarni, A. K. Naik, and N. S. Nagori, "A comparison of real valued transforms for image compression," International Journal of Engineering and Applied Sciences, vol. 6, no. 5, pp. 285-289, 2010.

[40] J. Oliver and M. P. Malumbres, "A new fast lower-tree wavelet image encoder," in Proceedings of the IEEE International Conference on Image Processing (ICIP '01), vol. 3, pp. 780-783, IEEE Signal Processing Society Press, 2001.

[41] H. Kondo and H. Kou, "Wavelet image compression using sub-block DCT," in Proceedings of the 9th IEEE International Conference on Networks, pp. 337-330, 2001.

[42] J. Akhtar and M. Y. Javed, "Image compression with different types of wavelets," in Proceedings of the 2nd Annual International Conference on Emerging Techonologies (ICET '06), pp. 133-137, November 2006.
[43] T. Tanaka, "Generalized subspace rules for on-line pca and their application in signal and image compression," in Proceedings of the International Conference on Image Processing (ICIP '04), pp. 1895-1898, October 2004.

[44] C. Lv, Z. Liu, and Q. Zhao, "A flexible non-linear PCA encoder for still image compression," in Proceedings of the 7th IEEE International Conference on Computer and Information Technology (CIT '07), pp. 645-650, October 2007.

[45] J. E. Fowler, "Compressive-projection principal component analysis," IEEE Transactions on Image Processing, vol. 18, no. 10, pp. 2230-2242, 2009.

[46] V. Gaidhane, V. Singh, and M. Kumar, "Image compression using PCA and improved technique with MLP neural network," in Proceedings of the IEEE International Conference on Advances in Recent Technologies in Communication and Computing (ARTCom '10), pp. 106-110, 2010.

[47] S. Grgic, M. Grgic, and B. Zovko-Cihlar, "Performance analysis of image compression using wavelets," IEEE Transactions on Industrial Electronics, vol. 48, no. 3, pp. 682-695, 2001.

[48] Z. Xiong, K. Ramchandran, M. T. Orchard, and YA. Q. Zhang, "A comparative study of DCT- and wavelet-based image coding," IEEE Transactions on Circuits and Systems for Video Technology, vol. 9, no. 5, pp. 692-695, 1999.

[49] D. Zhang and S. Chen, "Fast image compression using matrix K-L transform," Neurocomputing, vol. 68, no. 1-4, pp. 258-266, 2005.

[50] B. S. Yarman, U. Guz, and H. Gurkan, "On the comparative results of "SYMPES: a new method of speech modeling", International Journal of Electronics and Communications, vol. 60, no. 6, pp. 421-427, 2006.

[51] Ü. Güz, H. Gürkan, and B. S. Yarman, "A new method to represent speech signals via predefined signature and envelope sequences," EURASIP Journal on Advances in Signal Processing, vol. 2007, Article ID 56382, pp. 1-17, 2007.

[52] H. Gurkan, U. Guz, and B. S. Yarman, "Modelling of electrocardiogram signals using predefined signature and envelope vector sets. Special Issue on Advances in Electrocardiogram Signal Processing and Analysis," EURASIP Journal on Applied Signal Processing, vol. 2007, Article ID 12071, pp. 1-12, 2007.

[53] H. Gürkan, U. Guz, and B. S. Yarman, "EEG signal compression based on classified signature and envelope vector sets. Special Issue on Bridging Technology Innovations to Foundations," International Journal of Circuit Theory and Applications, vol. 37, no. 2, pp. 351-363, 2009.

[54] U. Guz, H. Gurkan, and B. S. Yarman, "A novel image compression method based on classified energy and pattern blocks: initial results," in Proceedings of the 25th International Symposium on Computer and Information Sciences, 2010.

[55] A. N. Akansu and R. A. Haddad, Multiresolution Signal Decomposition, Academic Press, San Diego, Calif, USA, 1992.

[56] J. G. Proakis and D. K. Manolakis, Digital Signal Processing, Prentice Hall, New York, NY, USA, 4th edition, 2006.

[57] A. M. Neto, L. Rittner, N. Leite, D. E. Zampieri, R. Lotufo, and A. Mendeleck, “Pearson's correlation coefficient for discarding redundant information in real time autonomous navigation system," in Proceedings of the 16th IEEE International Conference on Control Applications (CCA '07), pp. 426-431, October 2007.

[58] C. Chinrungrueng and A. Suvichakorn, "Fast edge-preserving noise reduction ultrasound images," IEEE Transactions on Nuclear Science, vol. 48, no. 3, pp. 849-854, 2001.

[59] Image database, "USC Viterbi School of Engineering, Electrical Engineering Department, Signal and Image 
Processing Institute (SIPI)," http://sipi.usc.edu/database/database.cgi? volume $=$ misc.

[60] Z. Wang, A. C. Bovik, H. R. Sheikh, and E. P. Simoncelli, "Image quality assessment: from error visibility to structural similarity," IEEE Transactions on Image Processing, vol. 13, no. 4, pp. 600-612, 2004.

[61] Z. Wang, A. C. Bovik, and L. Lu, "Why is image quality assessment so difficult?" in Proceedings of the IEEE International Conference on Acoustic, Speech, and Signal Processing (ICASSP '02), vol. 4, pp. IV/3313-IV/3316, May 2002. 